\title{
Peritumoral Tissue Compression is Predictive of Exudate Flux in a Rat Model of Cerebral Tumor: an MRI Study in an Embedded Tumor
}

James R. Ewing1, 2, 3*, Tavarekere N. Nagaraja ${ }^{4}$, Madhava P. Aryal ${ }^{5}$, Kelly A. Keenan ${ }^{4}$, Rasha Elmghirbi1 ${ }^{13}$, Hassan Bagher-Ebadiann ${ }^{1,3}$, Swayamprava Panda' ${ }^{1}$, Mei Lu ${ }^{6}$, Tom Mikkelsen ${ }^{1,4}$, Glauber Cabral ${ }^{1}$, Stephen L. Brown ${ }^{7}$

${ }^{1}$ Dept. of Neurology, Henry Ford Hospital, Detroit, MI, 2Dept. of Neurology, Wayne State University, Detroit, MI, ${ }^{3}$ Dept. of Physics, Oakland University, Rochester, MI, ${ }^{4}$ Dept. of Neurosurgery, Henry Ford Hospital, Detroit, MI, ${ }^{5}$ Department of Radiation Oncology, University of Michigan Medical School, Ann Arbor, MI, ${ }^{6}$ Dept. of Public Health Sciences, Henry Ford Hospital, Detroit, MI, ${ }^{7}$ Dept. of Radiation Oncology, Henry Ford Hospital, Detroit, MI

*Corresponding Author: James R. Ewing; jewing1@hfhs.org

Grant sponsor: National Institutes of Health: MRI Biomarkers of Response in Cerebral Tumors; Grant number: R01 CA135329

Word count: 9800 , Including appendix, 9 figures in main body, 1 in appendix, 42 references, one Supplemental Material file.

Running Head: Peritumoral tissue compression and glioma exudate

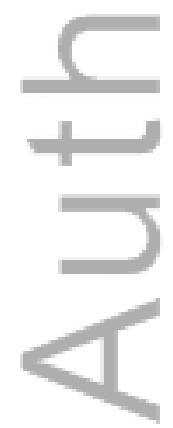

This is the author manuscript accepted for publication and has undergone full peer review but has not been through the copyediting, typesetting, pagination and proofreading process, which may lead to differences between this version and the Version of Record. Please cite this article as doi: 10.1002/NBM.3418

This article is protected by copyright. All rights reserved. 


\section{Abstract:}

MRI estimates of extracellular volume and tumor exudate flux in peritumoral tissue are demonstrated in an experimental model of cerebral tumor. Peritumoral extracellular volume predicted the tumor exudate flux.

Eighteen RNU athymic rats were inoculated intracerebrally with U251MG tumor cells and studied with dynamic contrast-enhanced MRI (DCE-MRI) approximately 18 days postimplantation. Using a model selection paradigm and a novel application of Patlak and Logan plots to DCE-MRI data, the distribution volume (i.e., tissue porosity) in the leaky rim of the tumor, and in the tissue external to the rim (the outer rim), was estimated, as was the tumor exudate flow from the inner rim of the tumor through the outer rim.

Distribution volume in the outer rim was approximately half that of the inner adjacent region $\left(\mathrm{p}<1 \times 10^{-4}\right)$. The distribution volume of the outer ring was significantly correlated $\left(\mathrm{R}^{2}=0.9\right)$ with tumor exudate flow from the inner rim. Thus, peritumoral extracellular volume predicted the rate of tumor exudate flux. One explanation for these data is that perfusion, i.e., the delivery of blood to the tumor, was regulated by the compression of the mostly normal tissue of the tumor rim, and that the tumor exudate flow was limited by tumor perfusion.

Key Words: Tumor Interstitial Volume, Interstitial Flow, Logan Plot, Patlak Plot, dynamic contrast enhanced MRI, DCE-MRI, tumor vasculature 
List of abbreviations: DCE-MRI, dynamic contrast enhanced MRI; CA, contrast agent; $v_{p}$, plasma volume; AIF, arterial input function; $\mathrm{K}^{\text {trans }}$, forward volume transfer constant; $\mathrm{v}_{\mathrm{e}}$, interstitial volume fraction; $V_{D}$, extracellular distribution volume; $k_{e p}$, reverse transfer constant; ROI, region of interest; SM, standard model; GLM, generalized linear model; AIC, Akaike information criterion; 2GE, dual-echo gradient-echo; LL, Look-Locker; TIFP, tumor interstitial-fluid pressure; PET, positron emission tomography; CT, computer-assisted tomography; H\&E, hematoxylin and eosin

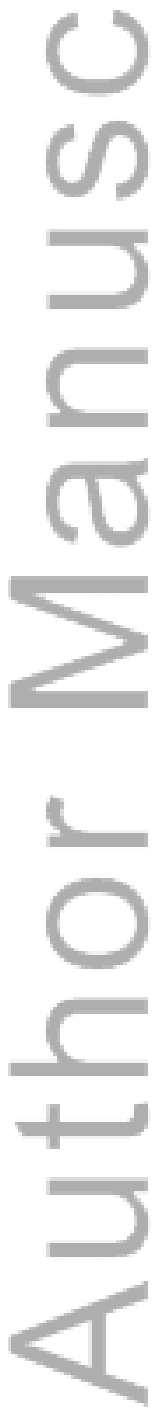

This article is protected by copyright. All rights reserved. 


\section{Introduction:}

Using animals implanted with a model U251 cerebral tumor and studied twice in a $24 \mathrm{~h}$ interval by dynamic contrast enhanced magnetic resonance imaging (DCE-MRI), an estimate of tissue extracellular volume fractions in both tumor boundary and the surrounding normal tissue will be presented. The outward flux of a contrast agent (CA) in interstitial tumor exudate in the tumor boundary will also be presented. Extracellular volume fraction and flux in the tumor boundary will be shown to be highly correlated.

An outward transport of CA may indicate increased tumor interstitial fluid pressure (TIFP) (1-4), which is associated with increased tumor aggressiveness (3). In order to noninvasively quantify TIFP, both the velocity of tumor fluid exudate and tissue fluid conductivity in the tumor boundary must be measured. It is the purpose of this paper to demonstrate methods for quantifying the extracellular distribution volume $\left(V_{D}\right)$ of $C A$ at the tumor edge and in its normal surround, and to estimate tumor exudate flux at the tumor boundary. $V_{D}$ is closely related to porosity $(\varphi)$ which in turn may be used, with appropriate calibration, to estimate tissue fluid conductivity (5).

The stepping-off point of this paper lies in a previous consideration of model selection and systematic errors in DCE-MRI studies (6), where it was shown (Figure 7 of reference (6)) in the rat model of cerebral tumor employed herein that, in the apparently normal tissue rimming the embedded tumor, model failure in the form of near-zero and even negative estimates of plasma volume $\left(\mathrm{v}_{\mathrm{p}}\right)$ typically occurred. The apparent cause of this model failure (Figures 1, 2, 3 of reference (6)) was the transport, via tumor exudate streaming, of the CA from the tumor interior to the voxels where the negative estimates of $\mathrm{v}_{\mathrm{p}}$ appeared.

Herein, a Logan Plot (7-9) is used to estimate the $V_{D}$ of CA in the tumor itself, and, uniquely, in the tumor surround. We have found (9) that a Logan Plot estimate of $V_{D}$ in the tumor is well correlated with cellular density and with a Standard Model (SM) (10) estimate of an equivalent quantity. Assuming that the border of the leaky

R3.10.E 
vasculature can be identified and associated with the tumor, it will be demonstrated that, a stable estimate of $V_{D}$ in the tumor surround can be generated by a Logan plot with an appropriately chosen input function. With the same assumptions it will also be shown that a Patlak Plot can estimate exudate flux through the tumor rim. Finally, the association of $V_{D}$ and flux at the tumor periphery will be demonstrated.

\section{Methods:}

Experimental methods, including the animal model, sample size, imaging methods, and post-mortem histopathology have been previously described $(9,11)$ and are further summarized in the Supplemental Material. All the animals presented herein have had their test-retest DCE-MRI studies analyzed for standard pharmacokinetic parameters, with results summarized and reported in reference (11). Thus, the experimental methods used herein will be only briefly summarized.

In a protocol approved by the Henry Ford Hospital institutional animal care and use committee, eighteen RNU athymic rats were inoculated intracerebrally with U251MG tumor cells and imaged approximately 18 days post-implantation. Tumors when imaged were about $5 \mathrm{~mm}$ in diameter. In order to establish test-retest variation in this animal model, two MRI studies were conducted for each animal, 24 hours apart.

DCE-MRI studies were performed at 7 Tesla. The DCE-MRI sequence used was a dual-echo spoiled gradient-recalled (2GE) sequence with a $60 \mathrm{~ms}$ TR and tip-angle of $27^{\circ}$. This allowed the direct calculation of changes in $\mathrm{T}_{2}{ }^{*}$ after the injection of $\mathrm{CA}$, and thus the construction of a time trace of change in $\mathrm{R}_{1}$ that was free of $\mathrm{T}_{2}{ }^{*}$ dephasing effects. Details may be found in reference (6). A three-slice set of $2 \mathrm{~mm}$ slices was centered over the tumor and a total of 150 image sets at intervals of $4 \mathrm{~s}$ were taken. Total run time was 10 minutes. Prior to the 2GE sequence, and immediately after, two Look-Locker (LL) sequences were run so that a voxel-by-voxel estimate of $\mathrm{T}_{1}$ in the tissue could be made pre- and post-CA administration. CA (Magnevist, Bayer Healthcare Pharmaceuticals, Wayne, New Jersey) 
bolus injection was performed by hand push at image 15. After a data-driven process for model selection and parametric estimation $(6,12,13)$, the slice with the largest tumor crosssection, usually the center slice, was selected for further analysis so that it might be assumed that the radial movement of CA was taking place in the plane of the image.

\section{Numerical and Statistical Methods:}

Using data from a series of LL-MRI studies performed before and after the 2GE DCE-MRI study, voxel-by-voxel maps of $\mathrm{T}_{1}$ pre- and post-contrast were generated $(12,14,15)$. After compensation for $\mathrm{T}_{2}{ }^{*}$ effects, the points of the DCE-MRI data before CA administration, and the last few points of DCE-MRI data, were calibrated against the LL $\mathrm{T}_{1}$ estimates performed pre- and post-study, thus creating two points, pre- and post-CA administration, that were used to generate and stabilize the running estimate of $\Delta R_{1}(t)\left(R_{1}=1 / T_{1}\right)$, which in turn was used to approximate the concentration-time curve of CA in the tissue (13).

A scaled radiological arterial input function (AIF) was used as the AIF in all studies $(6,9,16,17)$. The starting point of the AIF was aligned with the first appearance of CA in contralateral normal tissue. A global starting point was R3.7.C selected, usually one or two time points after the arrival of CA. For the calculation of the pharmacokinetic parameters of the SM, i.e. the extended Tofts $(10,18)$, or extended Patlak model, or in this paper, Model 3, the next 90 points (6 R3.1 minutes) of data were fitted by minimizing the sum squared error. For Logan plot calculations, because the Logan plot depends on the asymptotic behavior of the CA in the tissue, the entire interval of indicator uptake and clearance after the starting point was considered, with the linear last portion of the curve used to estimate $V_{D}$.

Model Selection: 
Model selection in this experimental paradigm (DCE-MRI in a rat model of cerebral tumor) has previously been presented $(6,12,13)$ and used to select regions of interest (ROIs) in recently published studies $(11,16,19)$. A full description of the model selection paradigm is presented in the supplemental material.

In the SM, there are at most three parameters to be estimated: the plasma volume $\left(v_{p}\right)$, the forward volumetric transfer constant ( $\left.\mathrm{K}^{\text {trans }}\right)$, and the reverse transfer constant $\left(\mathrm{k}_{\mathrm{ep}}\right)$. The often-reported interstitial volume fraction $\mathrm{v}_{\mathrm{e}}$ is computed as $\mathrm{K}^{\text {trans }} / \mathrm{k}_{\mathrm{ep}}$, although, because information in the data that pertains to the leakage of CA to the interstitium has to do with the first and second derivatives of concentration-time data, it is the rate constant $k_{e p}$ that is directly estimated. Consider the hierarchy of models that might prevail in the analysis of DCE-MRI data in the brain. For any one voxel, there are four possible descriptions, as follows: 0) the voxel contains little or no perfused tissue. Consequently, there is no detectable change in $\Delta \mathrm{R}_{1}$ after $\mathrm{CA}$ administration and all parameters are not different from 0.1 ) The vasculature in the voxel does not detectably leak CA across the period of observation: $\mathrm{v}_{\mathrm{p}} \neq 0, \mathrm{~K}^{\text {trans }}=\mathrm{k}_{\mathrm{ep}}=0$. This is the case in most normal brain. 2) The vasculature in the voxel detectably leaks CA, but there is little evidence of reflux from the tissue to the vasculature: $v_{p} \neq 0, K^{\text {trans }} \neq 0, k_{e p}=0$. and, 3 ) the vasculature detectably leaks $C A$, and there is evidence of reflux from the tissue to the vasculature. This is the full model described by the $S M, v_{p} \neq 0, K^{\text {trans } \neq 0}, k_{e p} \neq 0$. These models are numbered 0 through 3 , to reflect the number of parameters estimated. The models are nested, both physiologically and mathematically, so they can be compared via an F-statistic $(6,13)$, with the higher-order model selected only if it is a significantly better explanation of the data than the lower-order model.

In each voxel, one set ( 0 to 3 in number) of parameters is reported, with the choice of model driven by a model comparison performed via a noncentral F-test (20). In the brain, the maps produced are: a map of model selection, a nearly complete map of plasma volume, a partial map of $K^{\text {trans, }}$ and a smaller map of ve. See Figure 1 for an example of such maps. 
The element of this process that was most important to this paper was the delineation via model selection of the edge of the tumor.

\section{Patlak and Logan Plots:}

Patlak and Logan graphical approaches are central to inferences about model behavior. The key test employed in each graphical approach addresses the question as to what portion of the data can be linearized, when plotted as a Patlak (21), extended Patlak (22) an analysis that uses the SM - or Logan (8) plot. The portions of data where linearity prevails indicates those times in the experiments where certain critical assumptions hold.

In a region where a Patlak Model 2 plot is linear, indicator enters a last (sink) compartment and is trapped therein; only $\mathrm{v}_{\mathrm{p}}$ and $\mathrm{K}^{\text {trans }}$ can be estimated. In a region where a Patlak Model 3 plot is linear, an estimate of the full set of Model 3 parameters (including $\mathrm{ve}_{\mathrm{e}}$ ) is available. In a region where a Logan plot is asymptotically linear, the source and sink compartments reach an approximate equilibrium in concentration. Note that, while plasma concentration is considered the forcing function of the differential equation of the model (e.g. equation 1 in Logan et al (8), if another forcing function is available, the relationship still holds. For instance, if the forcing function is the concentration of indicator in an adjacent space, and that space is the source of indicator, the relationships outlined above still hold, and the portions of data where the critical assumptions hold will still plot as linear functions.

Statistical Methods for the Analysis of the Population Sample:

"Signal-to-noise" (S/N) in the first echo of the 2GE DCE-MRI data prior to CA administration was assessed by measuring the average image intensity of the whole brain in the middle slice of the slice pack, divided by the image intensity in the portions of the image where no tissue resided. In order to allow a better sense of conditions in a tumor of about $5 \mathrm{~mm}$ diameter, $\mathrm{S} / \mathrm{N} \pm$ S.D for the tumor itself, was also estimated, however, because the receiver employed a surface coil, the gradient in image intensity contributed to the variance of the measure of signal.. Note also that this is not the 
true $\mathrm{S} / \mathrm{N}$ in the complex time-domain data, but it is a convenient metric that is unimodal in the true $\mathrm{S} / \mathrm{N}$.

In the 18 studies, each with two measurements performed 24 hours apart, the parameters of interest were $V_{D}$ outside the tumor rim, and tumor exudate flux through the rim. Preliminary analyses examined differences in volume with t-tests and correlations between volume and flow with Pearson product-moment correlation coefficients. In order to account for the effect of correlated measures due to repeated studies, the final reporting analysis utilized a Generalized Linear Model (GLM) approach, as implemented in the library nlme (23) of the R Statistical Package (24). In comparing models produced by GLM, the Akaike Information Criterion (AIC) was used as a measure of goodness-of-fit; differences in the AIC were used to predict the probability that a model was descriptive of variation in the dependent variable.

\section{Results:}

$\mathrm{S} / \mathrm{N}$ in the DCE-MRI experiments was $\sim 30: 1 . \mathrm{S} / \mathrm{N}$ in the tumor itself was $\sim 35 \pm 4$.

See Figure 1, where the results of pixel-by-pixel estimates of DCE-MRI R3.12 model parameters in the brain of an athymic nude rat implanted with an U251 tumor are mapped. This animal study is chosen as typical of the 18 studies summarized in this Results section, and will be used throughout to illustrate the methods employed in the analysis of the full set of studies. A second study is shown in the Supplemental Material. Shown left to right are estimates of $v_{p}, K^{\text {trans }}, v_{e}$, and Model selection, where model 1 estimates only $v_{p}$, model 2 estimates $K^{\text {trans }}$ and $v_{p}$, and Model 3 estimates $v_{e}, K^{\text {trans }}$, and $v_{p}$. For reasons that will be explained below, the largest slice in the tumors in our animal model nearly always showed regions with near-zero or negative estimates of $v_{p}$ in the Model 2 regions. For instance, in Figure 1, the map of $v_{p}$ shows a nearly complete ring of negative estimates in the Model 2 region outside the tumor. Since they imply a failure of conservation of mass, these estimates are clearly artifacts and point 
to a model failure, but an insight into the source of these artifacts suggests a method for estimating the distribution volume of CA in the mostly normal tissue outside rim of the tumor.

First, it should be demonstrated that the CA escapes the boundaries of the tumor. An examination of the typical histology of the U251 cerebral tumor (the hematoxylin and eosin stained section of Figure 2 of reference (6), of Figure 7s of the Supplemental Material, and of Figure 2 herein) shows that, on the scale of the DCE-MRI image sets, where the pixel sizes are about 250 microns, the edge of the tumor is confined to a single pixel. The post-contrast $\mathrm{T}_{1}$-weighted images in reference (6), in

R.3.6A and C Figure 2 herein, and in Figure 7s of the Supplemental Material, which were acquired about 15 minutes after CA injection, show a bright rim of contrast around the central lesion; the DCE-MRI data in reference (6) show that CA leaks from the tumor across the time of the experiment, and, since the area of contrast enlarges radially R3.13 over time, is transported (by advection and/or diffusion) into the surrounding normal tissue, entering a region of mostly, or entirely, normal parenchyma where the blood-brain barrier restricts its reabsorption to the vasculature.

Notice the three pixels in Figure 1 that are marked by an arrow and three small green dots placed on the map of model selection. Figure 3 shows the change in $\Delta \mathrm{R}_{1}$ with time in the three linearly adjacent voxels, starting in the Model 3 region and proceeding outward in the Model 2 region. Two types of plot appear, with normally plotted data and curve fits in the left-hand figure, and Patlak-plotted data in the right-hand figure. In both figures, the three traces of concentration-time data, correspond top to bottom, to right to left points in Figure 1. The top trace is $\Delta \mathrm{R}_{1}$ data from pixel 1 , the Model 3 voxel; the middle trace is data from pixel 2 the first Model 2 voxel outside the inner Model 3 region, and the bottom trace is data from pixel 3, the next voxel out.

This article is protected by copyright. All rights reserved. 
Presumably, the tumor is the major source of CA seen in normal tissue in Figure 2. Consider top left region of interest (ROI) shown in Figure 4, defining the outer limit of the Model 3 region, outside of which is a region of predominantly Model 2 behavior with near-zero or negative estimates of $\mathrm{v}_{\mathrm{p}}$. Figure 5, left, displays the Patlak- and extended Patlak plots of concentration-time data in this inner rim ROI, demonstrating that Model 3 provides a better explanation of the behavior of the data than Model 2 because the Model 3 plot is linear while the Model 2 plot is concave. The F-statistic, with a value R3.15 of 725, yielding a vanishingly small probability that the errors of the Model 2 fit and the Model 3 fit were drawn from the same distribution, confirms this judgment. SM estimates of $\mathrm{v}_{\mathrm{p}}, \mathrm{v}_{\mathrm{e}}$, and $\mathrm{K}^{\text {trans }}$ in this inner-rim ROI are $1.1 \%, 10.8 \%$, and $3.0 \times 10^{-2} \mathrm{~min}^{-1}$, respectively. The Logan plot estimate of $V_{D}$ is $11.4 \%$. These estimates reflect the typical physiology of this model of cerebral tumor, with fairly high vascular permeability, and a decreased distribution volume fraction relative to the typical $20 \%$ of normal brain tissue $(25,26)$.

If a three-pixel-wide ROI (Figure 4, top right) is formed outside the inner ring defined by the edge of the Model 3 region the (Model 2) Patlak plot of Figure 6 is obtained. This plot has an evident linearity, a positive slope, a negative intercept, and a convex curve connecting the linear part of the curve to the start of the data. An examination of the methods of plotting the Patlak and extended Patlak plots shows that a convexity in the Patlak plot can only be obtained if $\mathrm{k}_{\mathrm{ep}}$ is negative, a clearly nonphysical situation that violates the conservation of mass, and/or the assumption of temporal stability. Nevertheless, the linear portion of the Patlak plot indicates that CA is entering the region, and is not being reabsorbed by the source. It also appears that the normalized standard radiological AIF to the outer region becomes approximately correct as the experiment proceeds, but that the input to this compartment lags the input of R3.7.D the adjacent compartment. These observations suggest that CA enters the ROI from the adjacent inner surface, and does not re-enter the source compartment in an appreciable amount during the course of the experiment. Note that, without a model selection 
paradigm with its two-parameter fit resulting in a negative $\mathrm{v}_{\mathrm{p}}$, in a three-

parameter SM analysis, $\mathrm{v}_{\mathrm{p}}$ and $\mathrm{K}^{\text {trans }}$ would be positive, and $\mathrm{k}_{\mathrm{ep}}$ negative, again signaling a model failure of standard pharmacokinetic modeling.

Consider a thin outer ROI drawn immediately adjacent to, and outside, the ROI of the edge of the Model 3 region. This ROI, a single pixel wide, is shown as the middle panel of Figure 4. Assume that the outer ring does not contain significantly leaky vasculature, and that the inner ring supplies the thin outer ring with the majority of its CA. The Logan plot of the CA concentration in the inner ring (Figure 5 Right) shows that a transvascular equilibrium occurs where the Logan plot becomes linear, after $100 \mathrm{~s}$, the $25^{\text {th }}$ point post-CA administration. That is to say, the CA concentrations in plasma and interstitial fluid are approximately equal after the $25^{\text {th }}$ point. Thus, it is plausible that, rather than using the arterial input function as an input to the outer ring, the CA concentration in the inner ring can be used as an input function, with the assumption that all the CA in the outer rim is obtained from the inner rim. It is assumed that the vascular R3.18 volume of the normal contralateral caudate putamen is $1 \%$. Since extravascular and intravascular CA concentrations have equilibrated, the extravascular concentration, i.e. the concentration of the interstitial fluid, is that of the blood. The trace of concentration in the inner ring is then normalized to be 100 times that of the trace of concentration in the (presumably non-leaky) contralateral caudate putamen. This is equivalent to requiring that the extravascular concentration of $\mathrm{CA}$ in the inner rim equilibrates with the intravascular concentration by the $25^{\text {th }}$ point post- CA injection, and that the vascular concentrations of CA do not substantially influence the behavior of the concentration-time studies under consideration. The biases introduced by vascular concentrations are examined in the Appendix.

The Logan plot of Figure 7 shows the result of these procedures. The plot becomes linear after the $97^{\text {th }}$ point post-contrast; fitting the last 35 points yields a slope of 0.0643 , or an estimate of $V_{D}$ of $6.4 \%$. Contrariwise, the Logan plot of this data, but using the estimate of 
CA plasma concentration versus time that resulted in the Logan plot of Figure 5 is shown in the insert of Figure 7. This graph yields a nonsensical result because the asymptotic behavior of the plot demonstrates a near-infinite slope, and thus R3.20 cannot estimate distribution volume in the tissue. This is suggestive of the inner rim being the source of CA for the outer rim.

R3.21

Continuing with the practice of using the rim of the tumor as a source of CA in the adjacent outer voxels, consider the wide ROI shown in the top right-hand image of Figure 4. This ROI is 2 to 3 pixels wide and contains essentially all of the region outside of the tumor into which the CA leaks. Thus, it satisfies the requirements of the Patlak (Model 2) model, in that it is a final compartment in which no return to the source compartment takes place. Figure 8 shows the resulting Patlak plot, which equilibrates very quickly, and appears to be linear for almost the entirety of the experiment. The slope of the plot, $2.9 \times 10^{-3}[\mathrm{ml} / \mathrm{ml}-$ $\mathrm{min}$ ] in this case, should give an estimate of the rate of transfer of the CA between the inner rim and the outer sink. When normalized to the volume of the wide ROI and the area of the interface between the inner and outer ring ROIs (see the Appendix, Case 2), it yields an estimate of CA flux between the two compartments.

The compartment size (of the wide outer ROI) into which the CA flows is 134 voxels, and there are 34 voxels on the rim of the tumor. Pixel edge sizes are $32 / 128 \mathrm{~mm}=1 / 4 \mathrm{~mm}$; slice thickness is $2 \mathrm{~mm}$. The average flux per voxel is thus about $4.5 \times 10^{-2} \mu \mathrm{l} / \mu \mathrm{m}^{2} \mathrm{~s}$. Taking the estimates of interstitial volume fraction to be $6.43 \times 10^{-2}$ for the outer ring, and $1.14 \times 10^{-1}$ for the inner ring, this rate of flux yields an estimate of fluid velocity in the interstitial space in the outer ring of $0.70 \mu \mathrm{m} / \mathrm{s}$, and for the inner ring, an estimate of $0.37 \mu \mathrm{m} / \mathrm{s}$. These velocities are in the range of other estimates of tumor velocity, i.e., less than 1 $\mu \mathrm{m} / \mathrm{s}$. See, for instance, Munson's (27) Table 1, also Pishko et al (28).

Table 1 summarizes the results of 34 studies in 18 animals. Sixteen of these studies were repeated studies; in Table 1 the first and second of the studies are labeled "pre" and "post," 
respectively. In the three parameters measured, there appeared to be no systematic differences between estimates in the two studies. That is to say that the two studies did not differ significantly in their sample means between study 1 and study 2 in the estimates of any parameter, nor did the paired differences show a trend by a paired t-test. The smallest $p$-value for any of the paired t-test comparisons was $\sim 0.3$ for the paired estimates of exudate flux; the smallest $p$-value for any of the tests on the sample means of study 1 and study 2 was $\sim 0.17$, again for flux.

The sample mean of $V_{D}$ in the inner ring ROI (tumor) was about 15\%; in the (presumably normal) outer ring, it was about $10 \%$. These estimates, particularly that of the outer ring, are smaller than the measured distribution volume of normal brain, which is about $20 \%$ across a wide variety of mammalian species $(25,26)$. There were distinct differences between the estimated $V_{D}$ of the inner ring region and that of the immediately adjacent outer ring. In 33 of 34 studies, the distribution volume in the outer ring ROI was smaller than its immediately adjacent inner ring ROI, and the one case where the difference was reversed was that of the second-smallest tumor cross-sectional area in the sample. In an initial analysis of parameters, the estimate of distribution volume in the inner ROI was significantly larger than that of the immediately adjacent outer ring in both study 1 ( $\mathrm{p}<$ $\left.4 \times 10^{-4}\right)$ and study $2\left(\mathrm{p}<1 \times 10^{-4}\right)$. When calculating the difference between the inner and outer ring distribution volumes, there was no significant difference $(p=0.63)$ between study 1 and study 2. A GLM analysis confirmed these findings: the outer ring ROI estimate of $V_{D}$ was highly predictive of the inner ring's estimate of $V_{D}\left(p<1 \times 10^{-4}\right)$. A regression slope of 0.528 demonstrated that the outer ring $V_{D}$ was usually about half that of the inner ring $V_{D}$. The intercept of this relationship was nonzero (intercept $=0.021, p \sim 0.043$ ), raising the possibility that some positive residual difference might be expected between the tissue of the inner (mainly tumor) and outer (mainly normal) ROIs. These analyses present a picture of a relatively porous inner ring of tissue that was mainly tumor, and a compressed outer ring of mainly normal tissue, in which it might be expected that the 
normal tissue would actually have a greater distribution volume than the tumor, absent the compressive forces placed on the outer ring by the tumor and the gradient of interstitial fluid pressure that typically exists in the rim of embedded tumors (29).

See Figure 9. When the estimated flux from the inner ring ROI to the outer expanded ROI was considered in relation to the estimated $V_{D}$ of the thin outer ring, a very significant relationship was demonstrated. An $\mathrm{R}^{2}$ of $0.9(\mathrm{~N}=34)$ calculated by a simple regression implied that all but a small amount of the variation in flux was described by the compressed distribution volume of the outer ring. In a GLM analysis for repeated measures, a regression coefficient of 0.740 was demonstrated: $V_{D}$ in the outer ring predicted $\left(\mathrm{p}<10^{-5}\right)$ the exudate flux through the outer ring of compressed tissue. The intercept of the regression, -0.011 probably differed from $0(p=0.049)$. The distribution volume of the inner ring was also a strong predictor of flux $\left(\mathrm{p}<10^{-4}\right)$, but a comparison of the two models via the AIC indicated that the outer rim $V_{D}$ was a vastly better predictor than the inner ring $\mathrm{V}_{\mathrm{D}}\left(\triangle \mathrm{AIC}=-45.5, \mathrm{p}=1.7 \mathrm{X} 10^{-20}\right)$. A multivariate GLM analysis that included $V_{D}$ of the inner ring showed no advantage to the inclusion of that parameter in the model $(\triangle \mathrm{AIC}=-8.9$ ). Thus, the estimated distribution volume in the rim of the normal tissue bounded by the tumor in itself appears to be sufficient to predict the rate of tumor exudate flux through the rim of the tumor in this model.

\section{Discussion:}

In an animal model of an embedded cerebral tumor, if its rim can be identified, a noninvasive estimate of extracellular space is available, both in the region of leaky vasculature, and in the immediate boundary of that region. If its rim of can be identified, an estimate of the exudate flow across the boundary of the tumor is also available. In this paper, using model selection to define the rim of the tumor, estimates of $V_{D}$ of CA (porosity) in the rim of the tumor and flux through that rim resulted in the porosity strongly and independently predicting the flux.

R3.10.D 
A key reservation in this finding is that it is assumed that the methods in play did in fact define the rim of the tumor, and that there was little or no vascular leakage in the ROI defined as the normal tissue outside tumor edge. A better model, given sufficient $S / N$, would assess both transvascular leakage and intervoxel transport. However, the information available in typical concentration-time curves is limited. It appears that two parameters is the highest number that the data demands. The suggestion that convection and vascular leakage be modeled simultaneously appears to demand at least four variables - $v_{p}, K_{\text {trans }}, k_{e p}$, and a directed inter-voxel flow. Even in the tumor itself, typical values of $R^{2}$ are 0.98 and above $(6,13)$, so, given the $\mathrm{S} / \mathrm{N}$ of a typical experiment, a stable estimate of four or more model parameters appears unlikely (6). It is possible that poroelastic modeling, given a starting-point estimate of distribution volumes (porosities), mechanical properties of tissue, and the time-dependent spatial concentrations of CA, might be employed to make a best-guess estimate of TIFP, intervoxel convection, and microvascular permeability (28,30-32). This appears to be a natural extension of the work presented herein, but one notes that the $\mathrm{S} / \mathrm{N}$ is limited in MRI studies of contrast transport, posing significant problems in extended modeling that uses dynamic MRI data for verification.

Although it has been of secondary interest in DCE-MRI, voxel-to-voxel transport of CA has been noted in cerebral tumors, sometimes as a source of artifact $(6,33)$, but also as a potential means of assessing tumor interstitial pressure (1-3) and/or evaluating tumor characteristics that affect the delivery of chemotherapy (30,34-36) and bias parametric estimates in DCE-MRI $(30,34)$. One experimental measure that affects all these considerations is the extracellular volume fraction, particularly because it can be expected that this quantity, equivalent to the porosity $(\varphi)$ of the tissue when the tissue is studied as a poroelastic medium $(31,37)$, is related to the fluid conductivity of the medium (5). In the absence of in-vivo experimental evidence, fluid conductivity is sometimes modeled as a constant across tissues (e.g. (30), and also the supplemental material of reference (38)), which may lead to biased results. 
In-vivo experimental estimates of porosity in tumors and normal tissues are relatively scarce. In humans, a PET study using ${ }^{76} \mathrm{Br}$-Bromide studied nine patients with cerebral tumors of varying grades, usually after bulk resection, and adjuvant therapy (39). ROIs were chosen to incorporate the highest concentration of radiotracer, so estimates of extracellular volume in the tumor, which ranged from $16 \%$ to $62 \%$ might be expected to be biased high. Estimates of extracellular volume in normal brain, about $20 \%$, were in agreement with classical measures in the brains of a wide variety of animal species $(25,26)$. While this in-vivo human study is interesting, the choice to inspect regions of highest ${ }^{76} \mathrm{Br}$ uptake, coupled to the limited intrinsic resolution of PET, and the treatments, particularly tumor resection, that the patients in this group had undergone, shed little light on tissue porosity and/or exudate flux at the rim of the tumor. Note also that a DCE-MRI study in patients with glioma (40) assessed interstitial volume in tumors as having two types of filling - the faster filling compartments had much lower values than the PET study, while the slower filling compartments tended to agree with the PET study, raising the likelihood that slowly filling necrotic regions were better sensed in the PET study.

An investigation in a rat model of cerebral glioma $(\mathrm{N}=4)$ employing a 9L gliosarcoma cell line performed DCE-MRI studies at 4.7T with similar timing and CA to the work herein, but with a different analysis (BOLERO (41)) that included the effects of water exchange. This study yielded much larger estimates of $v_{e}$ than those that appear herein, with porosity estimates of about 0.5. This large interstitial volume fraction seems implausible when viewing histology of the closely packed cells of a 9L cerebral tumor. A later paper (42) using a water exchange model (BALDERO) (43) with all three eigenvalues in play and a normalized AIF estimated $v_{e}$ in a U87 cerebral tumor model to be about 15\%, in fair agreement with this and other studies. We note that the estimate of $K^{\text {trans }}$ herein, and in other publications that use the SM to study both human and animal models of cerebral tumor $(6,11,13,16)$ are in fairly good agreement with those of CT perfusion estimates (44) that use a similarly sized CA with no dependence on water exchange. 
Some additional discussion is warranted by the concern that variation between the true AIFs in individual studies and the group-averaged AIF that was employed throughout the sample undermines the results of these studies. The most straightforward approach to this topic is to refer to the tables of reference (11). That work contains a statistical analysis of the 24 hour test-retest variation in the sample of animals presented in this paper. In Model 3 regions, paired differences were not significantly different from 0 for any of the model parameters, or for either of the summary statistics (mean, median). For $\mathrm{K}^{\text {trans }}$ and $\mathrm{V}_{\mathrm{e}}$ (Tables 2 and 3 ), the sample of paired differences displayed very small medians and means, less than $3 \%$ for $\mathrm{K}^{\text {trans }}$, and about $5 \%$ for $\mathrm{v}_{\mathrm{e}}$. The parameter $\mathrm{v}_{\mathrm{p}}$ showed higher sampling differences - about $15 \%$ in the mean, which might be expected of a parameter that, unlike $\mathrm{K}^{\text {trans }}$ and $\mathrm{V}_{\mathrm{e}}$, is sensitive to the high-frequency components of the AIF. Test-retest areas of Model 3 did not differ. Thus, the sample of animals used in this present paper doesn't present a picture of large variability in the parametric estimates of the Model 3 region. A summary of Model 2 parameters and areas also did not show large sampling mean differences. This implies that the underlying processes involved in the DCE-MRI studies were stable, and should alleviate concern about the influence of hand injection of CA.

There are sound physical reasons to explain the experiment's insensitivity to the details of the shape of the input function. The transfer of CA to the interstitium constitutes a low-pass filter - the components of the input function that are passed to the interstitium are the ones with long time constants. In the Model 3 regions (i.e., in the tumors) of both humans (13) and animals (6), $\mathrm{R}^{2}$ values of 0.98 and higher are typical for a 6 minute DCE-MRI analysis, so the low-pass filter explains almost the entirety of the signal behavior in the experiment. In both humans (13) and animals (6), typical rate constants for transfer of CA to and from tissue ( $\mathrm{K}^{\text {trans }}$ and $\mathrm{kep}_{\mathrm{ep}}$ ) in the tumor are $<0.1 \mathrm{~min}^{-1}$, meaning that the components of the input function that are important in Model 3 regions are those with time constants of about 10 minutes or longer. The most important element of the input function is the DC component; the short-term details of the shape of the input function do not 
contribute strongly to the fitting of the major component of the tissue response. In order to reliably estimate the DC component of the input function, we utilize the very useful $a$ priori knowledge that CA does not leak from the vasculature in normal tissue across the 6 minute time of the experiment (45), and that the plasma volume in the caudate putamen is approximately $1 \%$ (46). These matters are further explained in the Supplemental Material.

Figure 9, displaying 32 samples in 16 animals, appears to describe a major effect in the physiology of an embedded cerebral tumor; about $90 \%$ of the variation is explained by one relationship. That a decreased distribution volume in the rim of a tumor so strongly predicts tumor exudate flux generates a puzzle in modeling. If one infers that tumor rim compression governs exudate flux to the practical exclusion of other effects, what then is the role of the T, or of the solid stresses generated by tumor R3.10.C growth? Measures of TIFP in this model (data not shown) are generally in the range of 5 to $15 \mathrm{~mm} \mathrm{Hg}$, and cellularity is also known to vary - Table 1 shows a factor of 4 in $V_{D}$ in the inner rim ROI. Since cellularity and $V_{D}$ co-vary (11), it is likely that tumor rim cellularity varied substantially, and along with that variation, tumor rim compression due to hypercellularity varied from animal to animal. Further, a number of constraints having to do with the nature of the input function in the dynamic models were placed on the analysis of data, probably introducing a variation in those animals whose input function deviated from the assumptions necessary for the analysis. Despite these other likely variations, it appears that one parameter, the distribution volume in the rim beyond the tumor, was predictive of the great majority of the variation in the rate of exudate flux from the tumor.

One explanation for these data is that perfusion, i.e., the delivery of blood to the tumor, was regulated by the compression of the tumor rim, and that the total exudate flow was limited by total perfusion. It has been demonstrated that TIFP is regulated by perfusion pressure (47). It can be reasoned that TIFP, exudate flow, and compression of the tumor rim all covary in a fashion that depends on the relative mechanical properties of the tumor and its 
surround. This paper is not the place to carry out a poroelastic analysis of the range of possibilities, but we note that, in many tumors, the rigidity of the extracellular matrix, and of the tumor itself, is greater than that of the normal tissue in which the tumor is embedded, and that modeling of solid stresses in tumors predict that "elevated radial and circumferential stress levels extend beyond the tumor by at least one tumor radius (31)." If that is the case with the U251 tumors studied in this work, then a picture is presented of a relatively incompressible, albeit tightly packed, tumor surrounded by a layer of relatively compliant tissue whose mechanical properties essentially regulate flow in to, flow out of, and interstitial fluid pressure within the tumor.

Note that $U 251$ tumors of the size that were studied, $5-8 \mathrm{~mm}$ diameter, typically have necrotic cores. Thus, these embedded tumors may not have had the central support that has been modeled in solid tumors $(31,38)$. It may be that, without that central support, the solid stress associated with proliferating cells assumes less importance than the solid stress associated with a pressure gradient that projects into the normal surround of an embedded tumor.

A number of fairly strong constraints have been placed on the analysis of data in this work. Many of these have to do with the nature of the input function to the system being studied. In estimating $K^{\text {trans }}, k_{e p}, v_{p}$, R3.10.E and $V_{D}$ in the Model 3 region, it was assumed that a group-averaged arterial input function would serve adequately for individual input functions. It was also assumed that the integrated tissue response in the (presumably) non-leaky caudate putamen would serve to scale the group-averaged input function (by a factor of 100). In the Model 2 region, it was assumed that no vascular leakage occurred, that the sole source of contrast was the adjacent inner region, and that the contrast concentration of the inner rim could also be scaled to the contrast of the caudate putamen. It was assumed that for the time of the 
experiment, all of the tumor exudate remained within the bounds of an extended ROI drawn around the inner rim, and also that, at some time after the equilibration of the inner rim's extra- and intra-vascular concentrations, the amount of indicator flowing into, and out of, the thin outer rim equilibrated. Many of these assumptions and constraints are reasonable, based on the linearities in the data analysis that tend to confirm them, and on the generation of an orderly set of inferences that, in their internal consistency, tend to support the assumptions. However, some caution is necessary; these observations should be replicated in other tumors and tissues, and, where practical, be compared with other measures of exudate flow.

We note the benefit of using the model selection paradigms that were employed in this effort. The boundary of the Model 3 region apparently serves as a discriminant between tumor and normal parenchyma, and provides a clear choice for the location of a driving function of tumor exudate flow. Model selection in the GLM analyses also helped to provide a clear picture of the relative strengths of the associations between measured parameters, and allowed a clear path to the inferred relationships between flow and $V_{D}$. A model selection procedure generated an observation, the negative values of $v_{p}$ in the Model 2 rim of the tumor, that led to the hypothesis that CA was streaming from the leaky inner rim to the non-leaky outer rim, and thence to the ability to estimate $V_{D}$ in the boundary region of the tumor.

These results prompt a cautionary comment about the costs of employing a priori knowledge in model fitting. It does seem to make sense in the analysis of DCE-MRI data to at least bound the search space to physically possible values, and non-negative constraints are almost universally employed in DCE-MRI. Note, however, that estimates that lie outside those bounds signal that a fundamental mismatch between model and measurement has occurred; the work herein would probably not have been generated if there had been a 
non-negative constraint on the parameter estimates. That said, it would be quite possible, if another reliable way to define the edge of the tumor could be described, to proceed to estimate tissue compression and flux in the boundary of the tumor. It appears that the information contained in that analysis constitutes a description of tumor physiology, and probably of changes in tumor physiology under treatment.

\section{Acknowledgements}

The authors thank Jun Xu for excellent technical assistance. The many discussions with Joseph D Fenstermacher as to the nature of the Patlak plot, the history of estimates of cerebral permeability in normal brain, and the possible sources of artifact in the model's parametric estimates are gratefully acknowledged. Research reported in this publication was supported by National Cancer Institute of the National Institutes of Health under award number R01CA135329. The content is solely the responsibility of the authors and does not necessarily represent the official views of the National Institutes of Health.

This article is protected by copyright. All rights reserved. 
Table 1

\begin{tabular}{|c|c|c|c|c|c|c|c|c|c|c|c|}
\hline & \multicolumn{3}{|c|}{ Dist. Volume, Inner Edge } & \multicolumn{3}{|c|}{ Dist. Volume, Outer Edge } & \multicolumn{2}{|c|}{ Inner-Outer } & \multicolumn{3}{|c|}{ Flux $\left[\mu \mathrm{l} / \mu \mathrm{m}^{2}-\mathrm{s}\right]$} \\
\hline & PRE & POST & $\Delta$ & PRE & POST & $\Delta$ & PRE & POST & PRE & POST & $\Delta$ \\
\hline JS80 & +1 & 0.0691 & & & 0.0404 & & & -0.0287 & & 0.0151 & \\
\hline JS81 & 0.0653 & 0.0731 & -0.0077 & 0.0484 & 0.0618 & -0.0134 & -0.0169 & -0.0113 & 0.0249 & 0.0344 & -0.0095 \\
\hline JS82 & 0.2003 & 0.1842 & 0.0160 & 0.1093 & 0.1122 & -0.0029 & -0.0910 & -0.0720 & 0.0575 & 0.0614 & -0.0039 \\
\hline JS83 & 0.1390 & 0.1842 & -0.0452 & 0.0973 & 0.1430 & -0.0456 & -0.0417 & -0.0413 & 0.0562 & 0.0726 & -0.0164 \\
\hline JS87 & 0.1679 & 0.1385 & 0.0294 & 0.1552 & 0.1067 & 0.0485 & -0.0127 & -0.0318 & 0.1097 & 0.0641 & 0.0457 \\
\hline JS91 & 0.1443 & 0.1460 & -0.0017 & 0.1017 & 0.1062 & -0.0045 & -0.0425 & -0.0397 & 0.0606 & 0.0588 & 0.0018 \\
\hline JS93 & 0.1827 & 0.1512 & 0.0315 & 0.0842 & 0.0976 & -0.0134 & -0.0985 & -0.0536 & 0.0509 & 0.0529 & -0.0020 \\
\hline JS101 & 0.0767 & 0.0921 & -0.0154 & 0.0498 & 0.0722 & -0.0224 & -0.0269 & -0.0199 & 0.0264 & 0.0349 & -0.0085 \\
\hline JS132 & 0.1065 & 0.1140 & -0.0075 & 0.0762 & 0.0643 & 0.0119 & -0.0304 & -0.0497 & 0.0487 & 0.0452 & 0.0035 \\
\hline JS134 & & 0.1677 & & & 0.0960 & & & -0.0718 & & 0.0539 & \\
\hline JS135 & 0.1335 & 0.1210 & 0.0125 & 0.1163 & 0.0669 & 0.0494 & -0.0173 & -0.0541 & 0.0957 & 0.0454 & 0.0504 \\
\hline JS162 & 0.1950 & 0.1379 & 0.0571 & 0.1833 & 0.1121 & 0.0712 & -0.0118 & -0.0259 & 0.1322 & 0.0817 & 0.0505 \\
\hline JS163 & 0.0381 & 0.0716 & -0.0334 & 0.0448 & 0.0438 & 0.0010 & 0.0067 & -0.0277 & 0.0350 & 0.0174 & 0.0176 \\
\hline JS180 & 0.2178 & 0.1956 & 0.0222 & 0.1560 & 0.1436 & 0.0124 & -0.0618 & -0.0520 & 0.1058 & 0.1070 & -0.0012 \\
\hline JS181 & 0.1393 & 0.1261 & 0.0132 & 0.0835 & 0.0913 & -0.0078 & -0.0558 & -0.0348 & 0.0479 & 0.0618 & -0.0139 \\
\hline JS195 & 0.1163 & 0.1751 & -0.0587 & 0.0789 & 0.1040 & -0.0251 & -0.0374 & -0.0710 & 0.0474 & 0.0777 & -0.0303 \\
\hline JS205 & 0.3011 & 0.2388 & 0.0623 & 0.1307 & 0.1468 & -0.0161 & -0.1704 & -0.0920 & 0.1002 & 0.0835 & 0.0167 \\
\hline JS207 & 0.2144 & 0.1597 & 0.0547 & 0.1309 & 0.1061 & 0.0248 & -0.0835 & -0.0536 & 0.0891 & 0.0658 & 0.0233 \\
\hline Mean & 0.1524 & 0.1414 & 0.0081 & 0.1029 & 0.0953 & 0.0042 & -0.0495 & -0.0472 & 0.0680 & 0.0599 & 0.0077 \\
\hline SEM & 0.0166 & 0.0110 & 0.0089 & 0.0102 & 0.0075 & 0.0077 & 0.0110 & 0.0050 & 0.0327 & 0.0215 & 0.0244 \\
\hline
\end{tabular}

This article is protected by copyright. All rights reserved. 
Figures

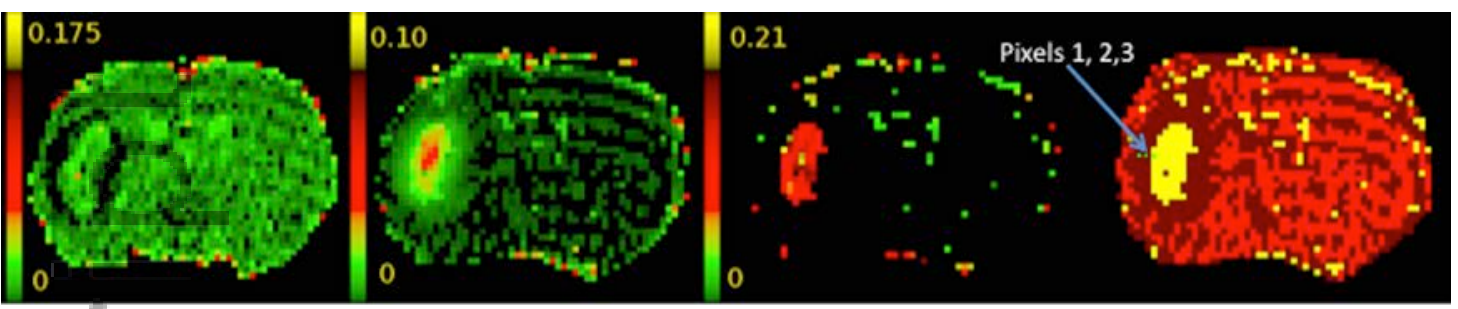

Figure 1: Parametric maps left to right: $\mathrm{v}_{\mathrm{p}}, \mathrm{K}^{\text {trans }}, \mathrm{v}_{\mathrm{e}}$ and Model selection. For the Model selection map, yellow is Model 3 acceptance, dark red is Model 2 acceptance, red is Model 1 acceptance. In order to better display the range of values, the map of $v_{p}$ was windowed to set negative values to 0 . Pixels 1, 2, 3 are marked with green dots and numbered from right to left, with pixel 1 placed in the margin of the Model 3 region, and pixels 2 and 3 in the Model 2 region in a line proceeding from pixel 1. Banding in the parametric maps and model selection is due to Gibbs ringing in the original 2GE data set.

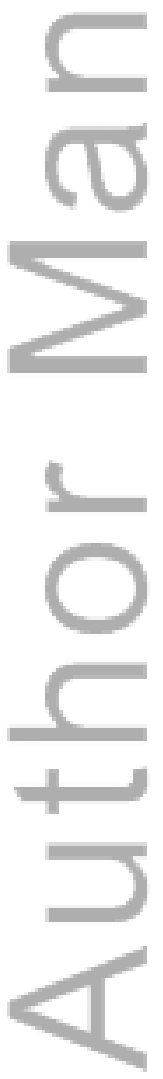



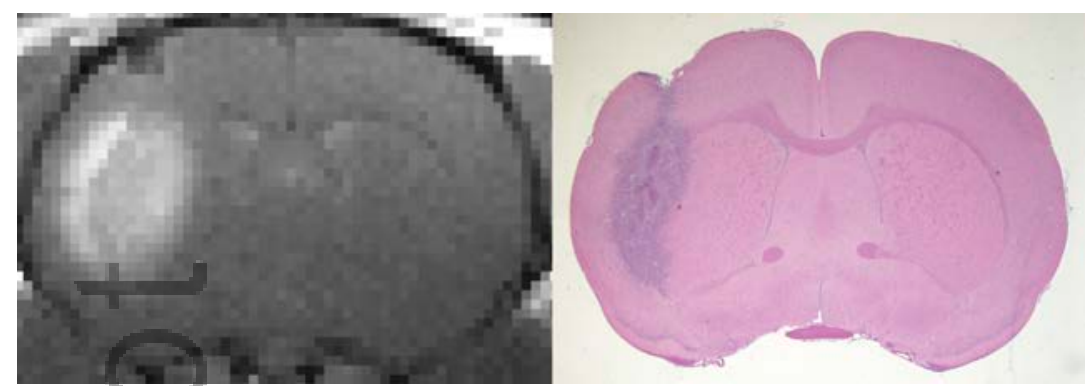

Figure 2: Left: post-contrast $\mathrm{T}_{1}$-weighted image In the animal of figure 1 . Right: H\&E staining of a centrally located tissue slice. The MRI image was acquired about 15 minutes after the injection of contrast agent. The central tumor mass is fairly well matched by the central but somewhat darker region of enhanced contrast, demonstrating the likely convection of the CA from the central lesion.

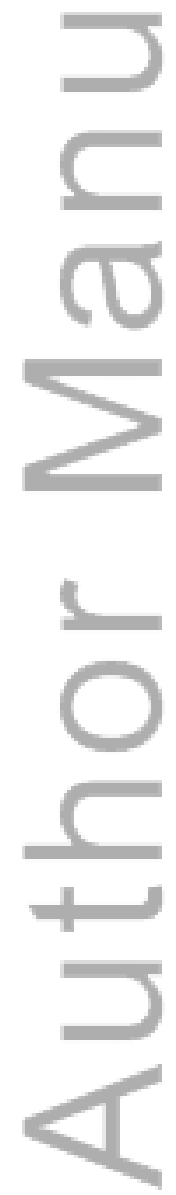

This article is protected by copyright. All rights reserved. 

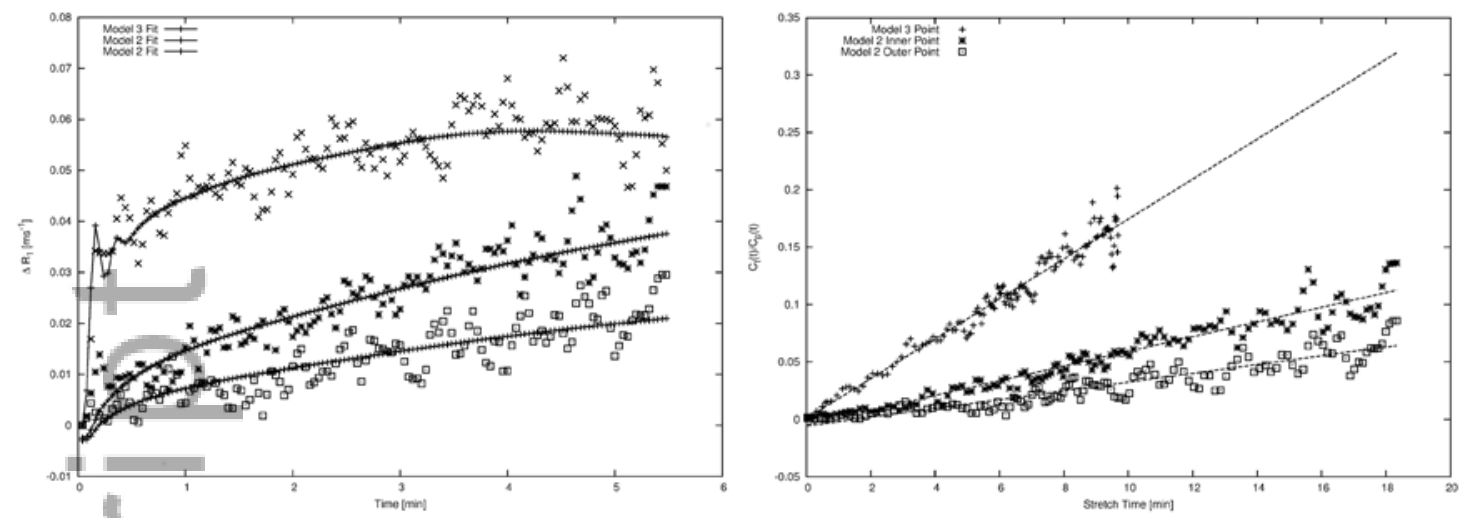

Figure 3: Left: Data and curve fits from the three marked pixels of Figure 1.

These three traces, top to bottom, correspond to right to left in Figure 1 - The top trace is $\Delta \mathrm{R}_{1}$ data from pixel 1 , the Model 3 voxel, the middle trace is data from pixel 2, the first Model 2 voxel outside the inner Model 3 region, and the bottom trace is data from pixel 3, the next voxel out. Right: linearized fits to the data showing the (Model 3) Extended Patlak fit (top) and the two (Model 2) Patlak fits of the data shown on the left.

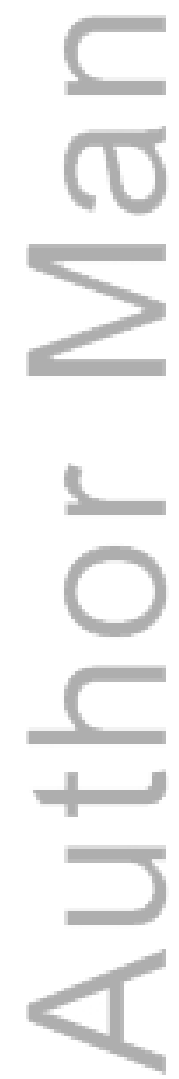




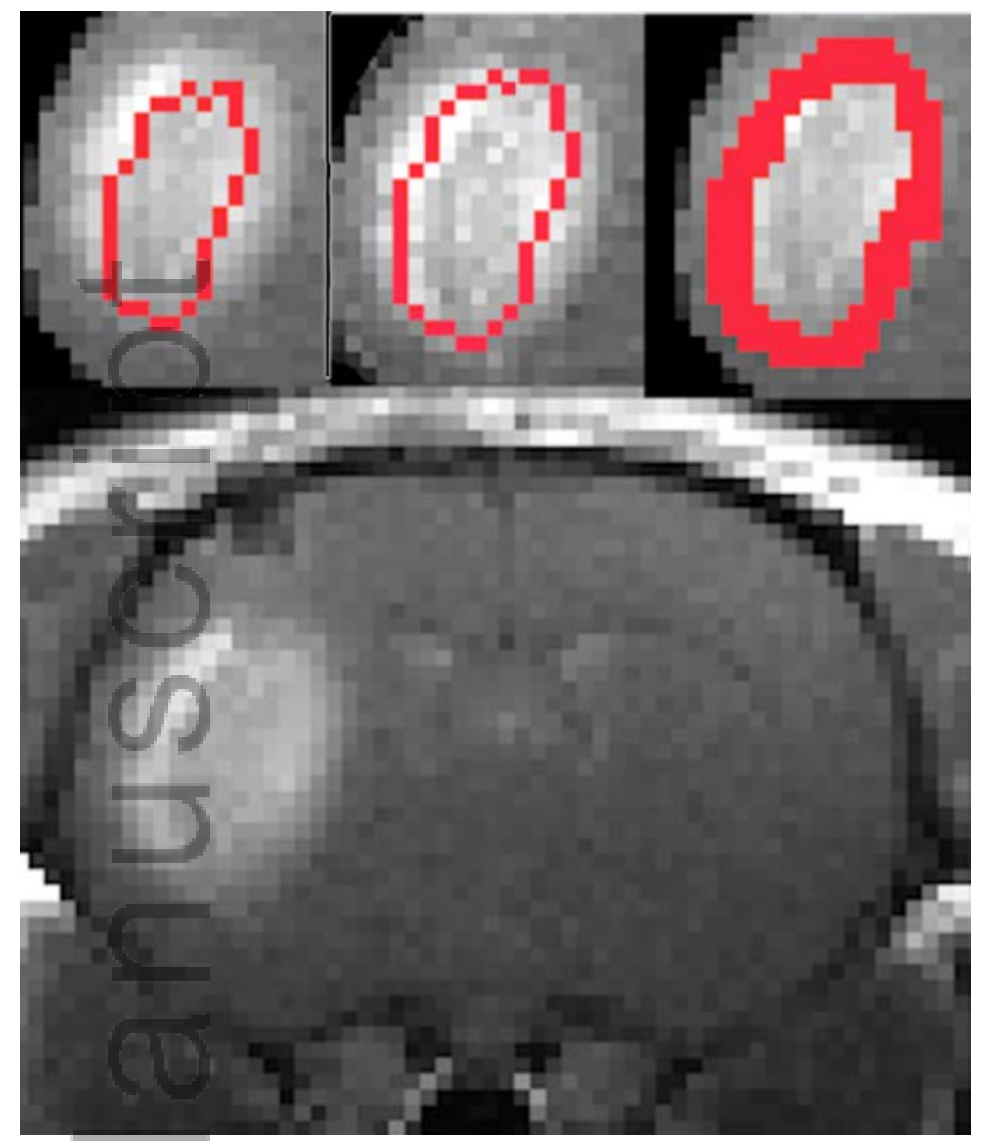

Figure 4: Three regions of interest in the animal under study. Left: an ROI defined as mainly Model 3, and inside the regions of negative estimate of $v_{p}$ in Figure 1. Middle: an ROI of single-pixel width, adjacent and outside of the ROI on the left, defined mainly by lying inside the regions of negative estimate of $v_{p}$. Right: the ROI of the middle panel, widened to include all of the regions of negative $v_{p}$ outside the inner rim defined by the right panel. The intent of these ROIs was to define a source region (left panel), an equilibrating region (middle panel), and a sink (right panel).

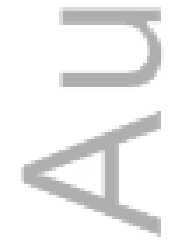



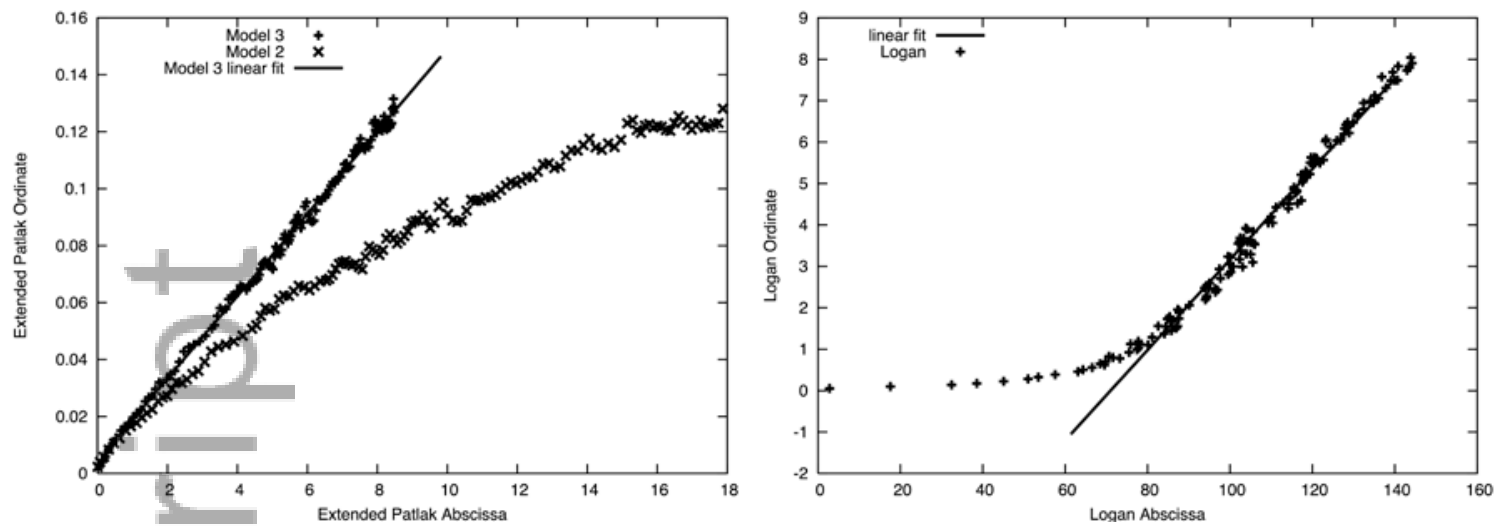

Figure 5: Left: Patlak (concave curve) and extended Patlak (linear curve) plots of response in the inner rim ROI (top left, Figure 4). Right: Logan plot of the same data. Both plots of the data employed the group-averaged estimate of the arterial input function, normalized to the plasma volume of the contralateral caudate putamen.

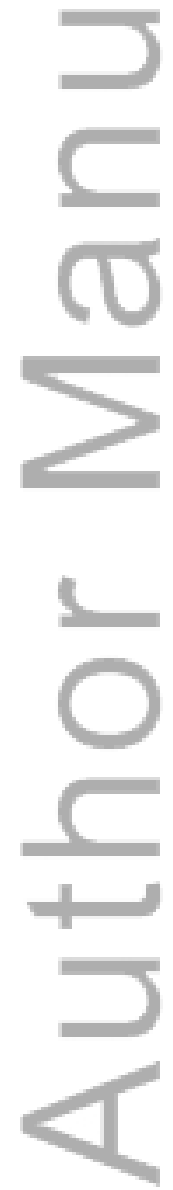




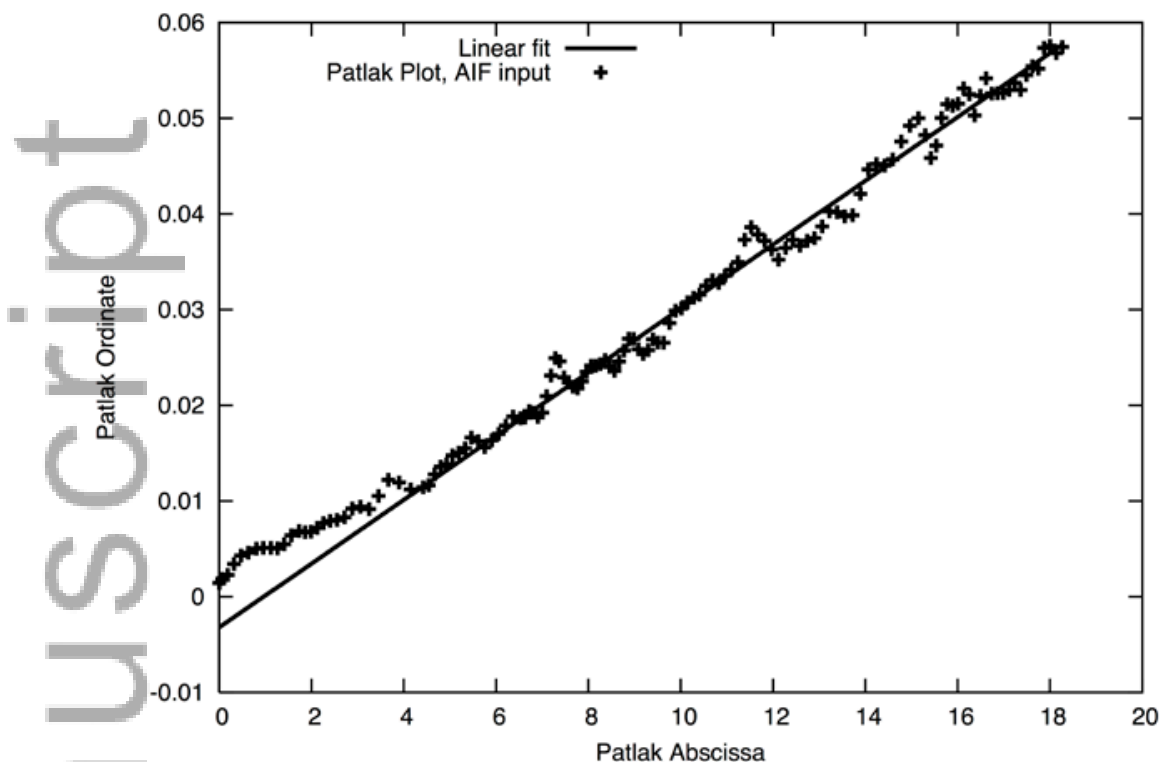

Figure 6: A Patlak Plot (Model 2) of the concentration-time data in the enlarged outer rim of the tumor. The input function employed was that of the group-averaged estimate of the arterial input function, normalized to the plasma volume of the contralateral caudate putamen, as in Figure 5. Note the linearity of the latter part of the curve, and the convex curvature of the early part of the curve, signaling a model failure.

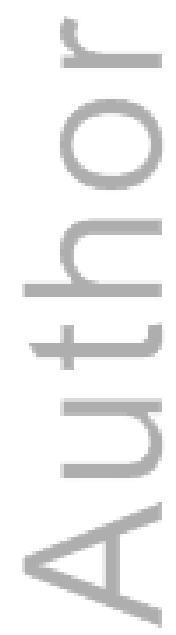

This article is protected by copyright. All rights reserved. 


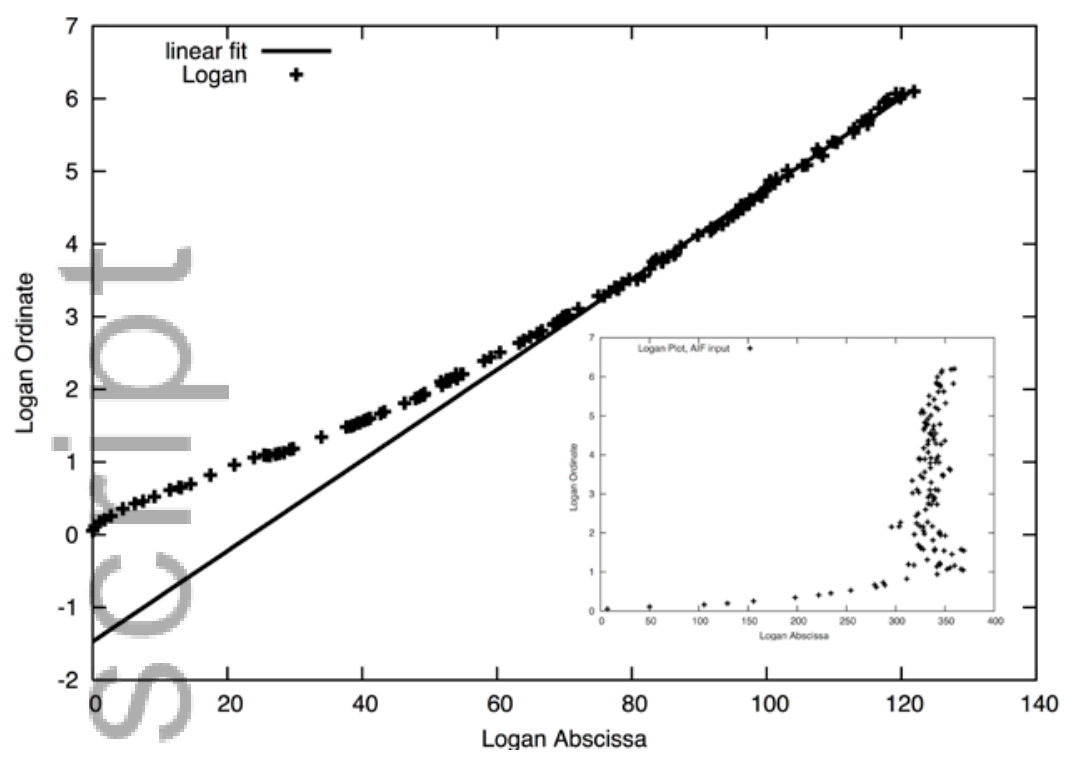

Figure 7: A Logan Plot of the concentration-time data in the thin outer rim of the tumor (central ROI, Figure 4). The input function employed was that of the normalized indicator concentration in the inner ROI. The equilibration point on the Logan Ordinate of about $80 \mathrm{~min}$ is evident, yielding an estimate of distribution volume of about $6.4 \%$. The same concentration-time data, plotted as a Logan plot, but using the estimated plasma concentration as an input function (Figures 5, 6) is plotted in the insert to demonstrate that the plasma concentration is not a viable input function for the concentration-time data of the thin outer rim, since the asymptotic behavior of the plot demonstrates a near-infinite slope, and thus cannot estimate distribution volume in the tissue. 


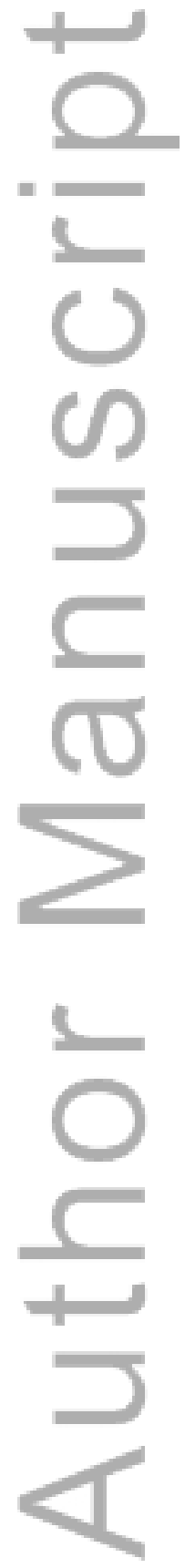

This article is protected by copyright. All rights reserved. 


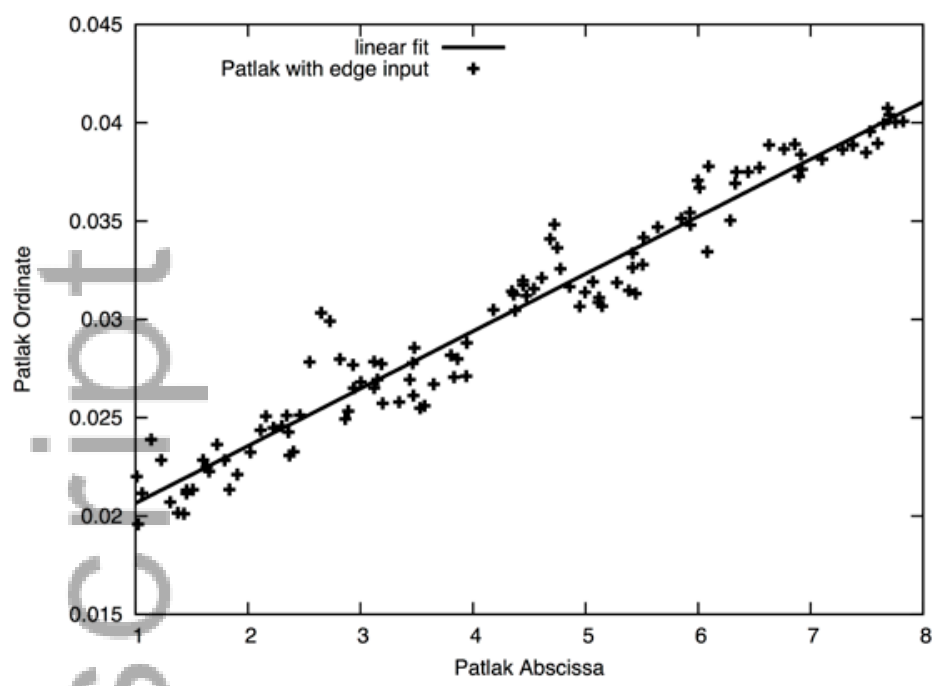

Figure 8: A Patlak Plot (Model 2) of the concentration-time data in the enlarged outer rim of the tumor (top right ROI in Figure 4), using as an input function the data of the innermost adjacent ring. The linearity of this portion of the curve is evident, thus supporting the assumptions of the model. The slope of the line yields an estimate of interstitial flow of tumor exudate.

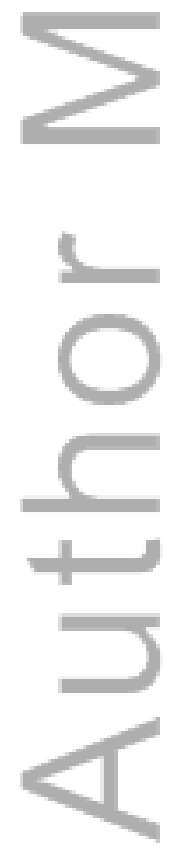




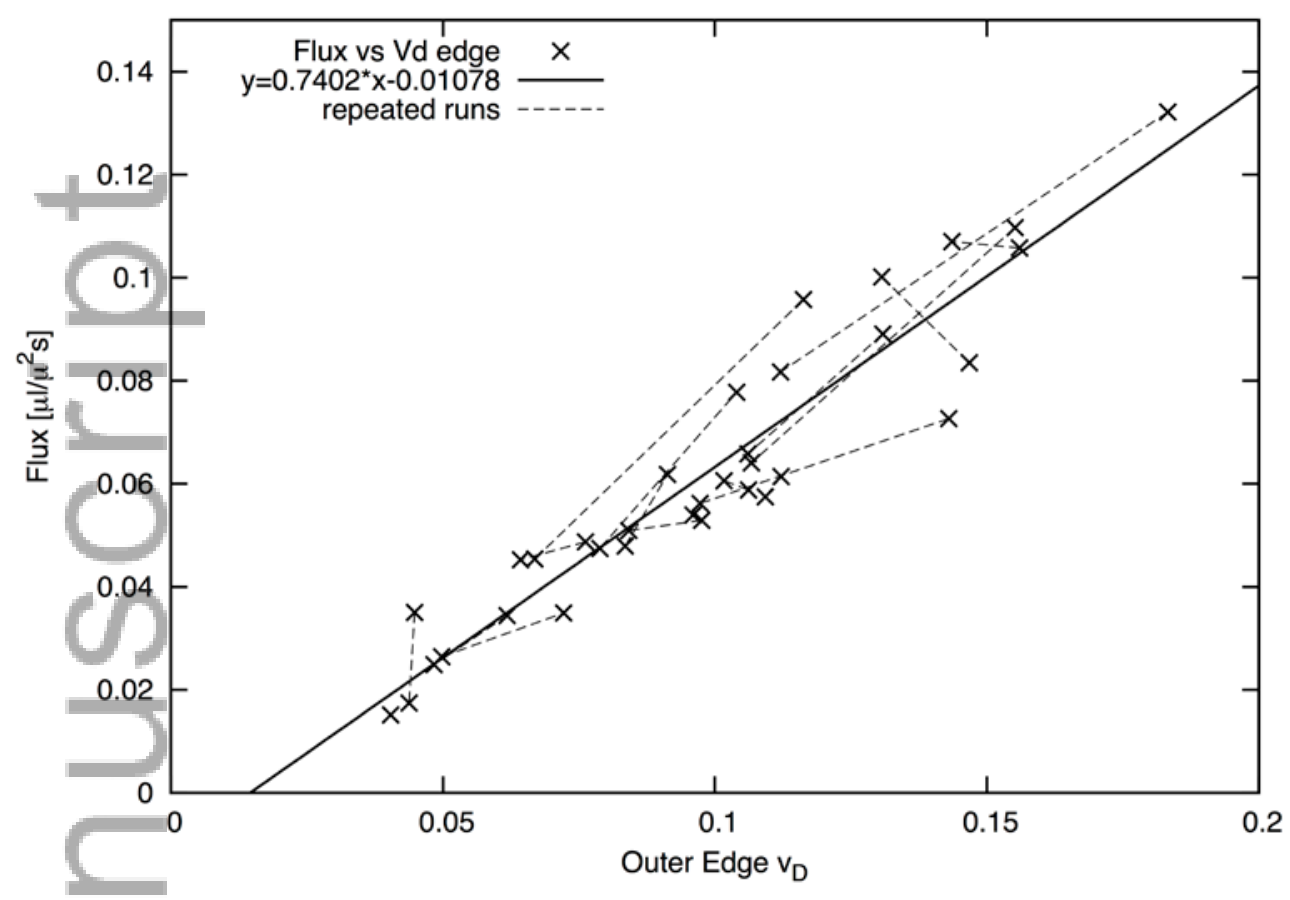

Figure 9: Exudate flux from the inner rim of the tumor to the outer ring in 18 animals. The 16 repeated studies are connected with dashed lines. This very significant co-variance implies that a knowledge of $V_{D}$ in the rim of the tumor will yield a remarkably precise prediction of exudate flow, regardless of other parameters such as TIFP and tumor porosity, which might otherwise be thought necessary for a prediction of the exudate flow rate.

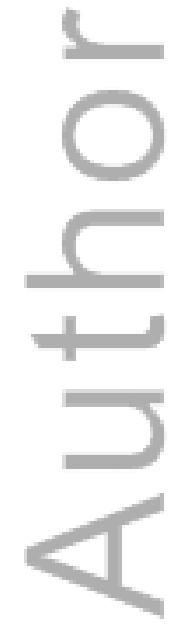

This article is protected by copyright. All rights reserved. 


\section{Appendix:}

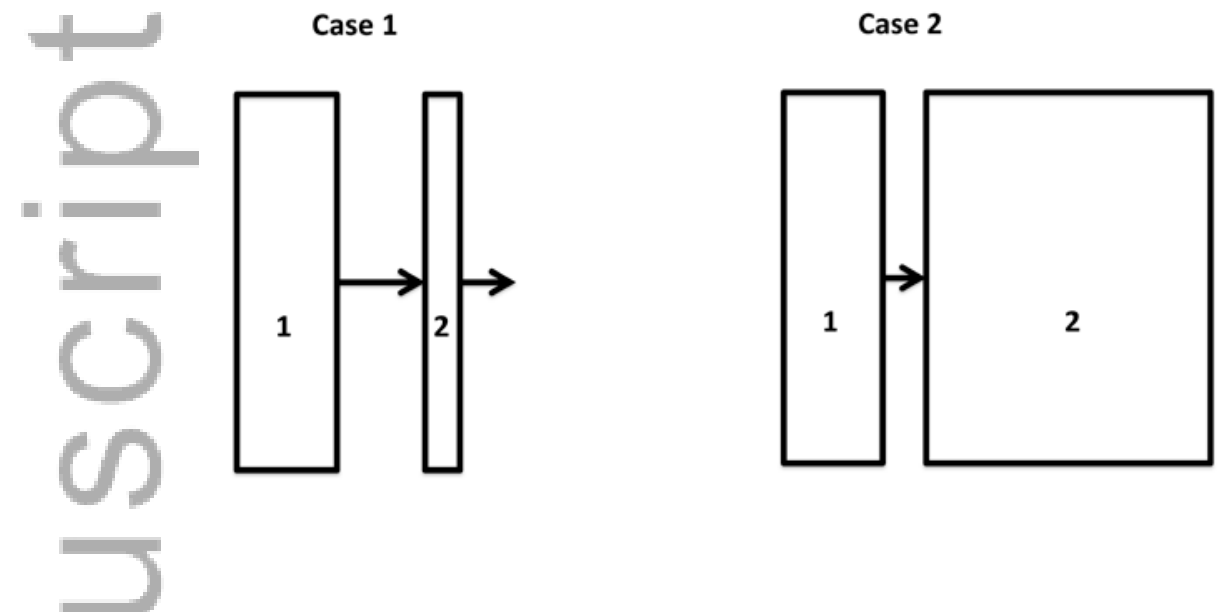

Figure A1: A two-compartment system. In case 1, contrast agent flows in to, and out of, compartment 2 . After some time $t_{1}{ }^{*}$, the vascular concentration of contrast agent in compartment 1 equilibrates with its interstitial concentration. After some time $t_{2}{ }^{*}$, the inflow and outflow of contrast agent in compartment 2 is approximately equilibrated. The equilibration is demonstrated by the Logan Plot of this data becoming linear. In case 2 , contrast agent flows into compartment 2, but is not cleared from the compartment. After some time $t^{\prime}$, a Patlak Plot (Model 2) becomes linear.

\section{Case 1: The Logan Plot in the Thin Rim:}

See Figure $\mathrm{A}$ 1, Case 1. In Case 1, fluid carries an indicator from compartment 1 to compartment 2 . The dimensions of compartment 2 are small compared to the velocity of the fluid so that, after a short time, the indicator begins to exit compartment 2 . When the slope of the input function to compartment 2 begins to change slowly, approximately the same amount of indicator is leaving the compartment as is entering it. This is an 
equilibrium condition that can be exploited to evaluate the distribution volume of compartment 2 .

Assume that there is no leakage from the microvasculature in compartment 2, that intravascular concentration of indicator can be ignored, and that indicator flows only from compartment 1 to compartment 2 , and thence to a sink. In a differential time interval, the change in the quantity, $\mathrm{Q}_{2}(\mathrm{t})$ of the indicator equals the difference between the quantity entering, and the quantity leaving the compartment:

$$
d Q_{2}(t)=F\left[C_{1 e}(t)-C_{2 e}(t)\right]
$$

where $\mathrm{F}$ is the flow from compartment 1 to compartment $2, \mathrm{C}_{1 \mathrm{e}}(\mathrm{t})$, and $\mathrm{C}_{2 \mathrm{e}}(\mathrm{t})$ are the concentrations of indicator in the extracellular interstitial fluid of compartment 1 and compartment 2 , respectively.

Integrating and dividing by the volume of the second compartment:

$$
\frac{Q_{2}(t)}{V_{2}}=\frac{F}{V_{2}}\left[\int_{0}^{t} C_{1 e}(\tau) d \tau-\int_{0}^{t} C_{2 e}(\tau) d \tau\right]+\frac{V_{2 p}}{V_{2}} C_{2 p}(t)
$$

Generates an expression in concentrations and specific flows:

$$
C_{2}(t)=f\left[\int_{0}^{t} C_{1 e}(\tau) d \tau-\int_{0}^{t} C_{2 e}(\tau) d \tau\right]+v_{2 p} C_{2 p}(t),
$$

Where, in compartment $2 \mathrm{~V}_{2}$ is the volume, $V_{2 p}$ is the plasma volume, $f$ is the specific flow $[\mathrm{ml} / \mathrm{ml}-\mathrm{min}]$, and $v_{2 p}$ is the fractional plasma volume. By convention, lower-case letters are associated with quantities that are normalized to volumes. Initially, consider only the interstitial concentrations (i.e., assume $v_{2 p}=v_{1 p}=0$, where $v_{i p}$ is the fractional plasma volume of the $i^{\text {th }}$ compartment). Continuing:

$$
\frac{C_{2}(t)}{f}=\int_{0}^{t} C_{1 e}(\tau) d \tau-\int_{0}^{t} C_{2 e}(\tau) d \tau
$$


Note that $C_{2}(t)=v_{2 e} C_{2 e}(t)$, where $v_{2 e}$ is the fractional volume of the interstitial space in compartment 2 and $\mathrm{C}_{2}(\mathrm{t})$ is the concentration of indicator in compartment 2. Dividing both sides of the equation by $\mathrm{C}_{2}(\mathrm{t})$ and transposing two terms yields an equation in the form proposed by Logan (8).

$$
=\frac{\int_{0}^{t} C_{2}(\tau) d \tau}{C_{2}(t)}=\frac{v_{2 e} \int_{0}^{t} C_{1 e}(\tau) d \tau}{C_{2}(t)}-\frac{v_{2 e}}{f}
$$

Under the assumption that the concentration of CA has equilibrated between inflow and outflow, and that $v_{1 p}=v_{2 p}=0$, a Logan Plot with $\frac{\int_{0}^{t} C_{2}(\tau) d \tau}{C_{2}(t)} \quad$ on the ordinate and $\frac{\int_{0}^{t} C_{1 e}(\tau) d \tau}{C_{2}(t)}$ on the abscissa will yield a straight line with a slope of $v_{2 e}$.

The interstitial concentration in compartment $1, C_{1 e}(t)$ is related to the tissue concentration, $C_{1}(t)$, via the relation $C_{1}(t)=v_{1 e} C_{1 e}(t)$. An estimate of $v_{1 e}$ is available through the Model 3 analysis, but there is a more reliable estimate of $\mathrm{C}_{1 \mathrm{e}}(\mathrm{t})$ that can be obtained through its behavior as described by a Logan Plot. In the case at hand, an examination of the Logan Plot of indicator concentration in the Model 3 region (i.e., compartment 1) at the edge of the tumor demonstrates that the exudate fluid in compartment 1 equilibrates with the plasma concentration. That is, the Logan Plot of compartment 1 becomes linear, with a slope of $V_{D}$, at times $t>t_{\mathbf{1}}^{*}$. Given this, the same tactic that applies to calibrating $C_{p}(t)$, i.e., scaling an input function to a large area of normal caudate putamen, with the assumption that $\mathrm{v}_{\mathrm{p}}$ in the caudate putamen is $1 \%$ of tissue volume, can be applied to the section of $\mathrm{C}_{1}(\mathrm{t})$ 
where $t>t_{1}$. This practice was followed throughout in estimating the interstitial space at the rim of the tumor.

It should be noted that only $C_{1}(t), C_{2}(t)$, and (indirectly) $C_{p}(t)$ are observable. The presence of a non-zero vascular volume, filled with $\mathrm{CA}$, introduces a bias into the result above.

Generally, since compartments 1 and 2 are adjacent, we can assume that the plasma concentrations in the two compartments are approximately equal. Thus $C_{1 p}(t)=C_{2 p}(t)=$ $\mathrm{C}_{\mathrm{p}}(\mathrm{t})$. The quantity of CA in compartments 1 and 2, respectively, are

$$
\begin{aligned}
& Q_{1}(t)=V_{1 p} C_{p}(t)+V_{1 e} C_{1 e}(t) \\
& Q_{2}(t)=V_{2 p} C_{p}(t)+V_{2 e} C_{2 e}(t)
\end{aligned}
$$

Dividing each by the volume of its compartment yield the following relationships between the measureable quantities $\mathrm{C}_{1}(\mathrm{t})$ and $\mathrm{C}_{2}(\mathrm{t})$, and the quantities of interest, $\mathrm{C}_{1 \mathrm{e}}(\mathrm{t})$ and $\mathrm{C}_{2 \mathrm{e}}(\mathrm{t})$ :

$$
C_{1 e}(t)=\frac{C_{1}(t)-v_{1 p} C_{p}(t)}{v_{1 e}} \text { and } C_{2 e}(t)=\frac{C_{2}(t)-v_{2 p} C_{p}(t)}{v_{2 e}}
$$

From Equations 2 and 5,

$$
\frac{C_{2}(t)}{f}=\frac{1}{v_{1 e}} \int_{0}^{t}\left[C_{1}(\tau)-v_{1 p} C_{p}(\tau)\right] d \tau-\frac{1}{v_{2 e}} \int_{0}^{t}\left[C_{2}(\tau)-v_{2 p} C_{p}(\tau)\right] d \tau+\frac{v_{2 p} C_{p}(t)}{f}
$$

Rearranging and collecting terms to generate an equation in the form of a Logan Plot,

$$
\frac{\int_{0}^{t} C_{2}(\tau) d \tau}{C_{2}(t)}=\frac{v_{2 e} \int_{0}^{t} \frac{C_{1}(\tau)}{v_{1 e}} d \tau}{C_{2}(t)}+v_{2 e}\left(\frac{v_{2 p}}{v_{2 e}}-\frac{v_{1 p}}{v_{1 e}}\right) \frac{\int_{0}^{t} C_{p}(\tau) d \tau}{C_{2}(t)}-\frac{v_{2 e}}{f}\left(1-\frac{v_{2 p} C_{p}(t)}{C_{2}(t)}\right)
$$


As before, the quantity $\frac{C_{1}(t)}{v_{1 e}}$ is estimated after the equilibration of plasma and interstitial concentrations in compartment 1 , and set equal to $\mathrm{C}_{\mathrm{p}}(\mathrm{t})$. This yields an equation in the form of a Logan Plot equation:

$$
\frac{\int_{0}^{t} C_{2}(\tau) d \tau}{C_{2}(t)}=\frac{v_{2 e} \int_{0}^{t} \alpha C_{1}(\tau) d \tau}{C_{2}(t)}+v_{2 e}\left(\frac{v_{2 p}}{v_{2 e}}-\frac{v_{1 p}}{v_{1 e}}\right) \frac{\int_{0}^{t} C_{p}(\tau) d \tau}{C_{2}(t)}-\frac{v_{2 e}}{f}\left(1-\frac{v_{2 p} C_{p}(t)}{C_{2}(t)}\right),
$$

Where $\alpha$ is the factor that scales the measured change in contrast in region 1 to the known concentration, that of the plasma, after time $t_{1}{ }^{*}$. Thus, a Logan Plot with
ordinate and $\frac{\int_{0}^{t} \alpha C_{1}(\tau) d \tau}{C_{2}(t)}$, on the abscissa, with $\alpha$ adjusted to equal $\underline{\int_{0}^{t} C_{2}(\tau) d \tau}$ $C_{2}(t)$ on the when the conditions of the assumption are met (flow of contrast in to, and out of compartment 2 is balanced), coupled with a slowly changing plasma concentration, will yield a straight line with a slope of $v_{2 e}\left(1+\frac{v_{2 p}}{v_{2 e}}-\frac{v_{1 p}}{v_{1 e}}\right)$. The interstitial volume fraction actions $v_{2}$ and $v_{1 e}$ are generally larger than their respective plasma volume fractions (typically a factor of 10 or more). Thus, assuming the worst case of $v_{1 p}=0$, with the other compartment ratio of relative volumes $\sim 1 / 10$, there is an upper limit on the error introduced by the presence of CA in compartments 1 and 2 of about $10 \%$. Generally speaking, since $v_{2 p} \approx v_{1 p}$, the error can be expected to be much smaller than $10 \%$.

\begin{tabular}{|l|l|l|l|}
\hline JS132 \\
\hline v1p & v2p & v1e & v2e \\
\hline
\end{tabular}




\begin{tabular}{|l|l|l|l|}
\hline 0.0110 & 0.01 (est) & 0.114 & 0.0642 \\
\hline
\end{tabular}

\section{Case 2: The Patlak Plot in the Wide Rim:}

In Case 2 above, no contrast exits from Compartment 2, considerably simplifying the treatment of the model. We ignore for the time being the plasma volume of compartment 1 , $\mathrm{V}_{1 \mathrm{p} .}=$

$$
\begin{aligned}
& d Q_{2}(t)=F C_{1 e}(t) d t \\
& Q_{2}(t)=F \int_{0}^{t} C_{1 e}(\tau) d \tau+V_{2 p} C_{2 p}(t)
\end{aligned}
$$

Normalizing to the volume of the second compartment and dividing both sides by $\mathrm{C}_{2 \mathrm{p}}(\mathrm{t})$ yields the Patlak (Model 2) equation.

$$
\frac{C_{2}(t)}{C_{2 p}(t)}=f \frac{\int_{0}^{t} C_{1 e}(\tau) d \tau}{C_{2 p}(t)}+v_{2 p}
$$

Remembering once again that $\mathrm{C}_{1 \mathrm{e}}(\mathrm{t})$ is known to equilibrate with the plasma concentration, we can say that after a time $t_{1}{ }^{*}$ the following relationship should hold:

$$
\frac{C_{2}(t)}{C_{1 e}(t)}=f \frac{\int_{0}^{t} C_{1 e}(\tau) d \tau}{C_{1 e}(t)}+v_{2 p^{2}}, t>t_{1}^{*} \text {, and a plot of } \frac{C_{2}(t)}{C_{1 e}(t)} \text { on the ordinate and } \frac{\int_{0}^{t} C_{1 e}(\tau) d \tau}{C_{1 e}(t)} \text { on }
$$

the abscissa will yield a straight line with a slope of specific flow [ $\mathrm{ml} / \mathrm{ml}-\mathrm{min}]$.

Note that this result pertains to specific flow normalized to the volume of compartment 2 . Since the size of compartment 2 varies across animals, another measure of transfer between the two compartments must be adopted in order to make comparisons. The total flow to compartment 2 is $\mathrm{fV}_{2}$, through a boundary of $\mathrm{N}_{2 b}$ voxels, where $\mathrm{V}_{2}$ is the volume of the second compartment, and $\mathrm{N}_{2 b}$ is the number of voxels in the inner boundary of compartment 2, i.e., the number of voxels in the thin outer rim. The volume of the second 
compartment is $V_{2}=N_{2 v} V_{v}$, where $N_{2 v}$ is the number of voxels in compartment 2, and $V_{v}$ is the volume of each voxel. Thus, if $A_{2 b}$ is the area of the inner surface of the outer ring ROI, a statistic that measures flux through the boundary can be calculated as: $f \frac{N_{2 v} V_{v}}{N_{2 b} A_{2 b}}$, in $\left[\mathrm{mm}^{3} / \mathrm{mm}^{2} \mathrm{~min}\right]$. This measure is reported as the flux across the boundary of the tumor.

Since the porosity of the thin outer rim can be estimated, another interesting measure that can be generated is the mean interstitial velocity of the tumor exudate. Given a measure of flux, $\phi$, the velocity of the tumor exudate in the interstitium is $\frac{\phi}{v_{e}}$.

We now turn to an evaluation of the errors introduced by ignoring the plasma concentration of CA in compartment 1. From Equations 5 and 8,

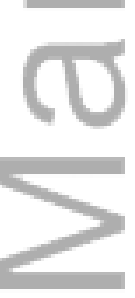

$$
\begin{aligned}
& C_{2}(t)=\frac{f}{v_{1 e}} \int_{0}^{t}\left[C_{1}(\tau)-v_{1 p} C_{1 p}(\tau)\right] d \tau+v_{2 p} C_{2 p}(t) \\
& C_{2}(t)=\frac{f}{v_{1 e}} \int_{0}^{t} C_{1}(\tau) d \tau-f \frac{v_{1 p}}{v_{1 e}} \int_{0}^{t} C_{1 p}(\tau) d \tau+v_{2 p} C_{2 p}(t)
\end{aligned}
$$

Dividing both sides by $\mathrm{C}_{2 \mathrm{p}}(\mathrm{t})$ to generate an equation in the form of Patlak graphical equation:

$$
\begin{aligned}
& \frac{C_{2}(t)}{C_{2 p}(t)}=\frac{f}{v_{1 e}} \frac{\int_{0}^{t} C_{1}(\tau) d \tau}{C_{2 p}(t)}-f \frac{v_{1 p}}{v_{1 e}} \frac{\int_{0}^{t} C_{1 p}(\tau) d \tau}{C_{2 p}(t)}+v_{2 p} \\
& =\int_{0}^{t} C_{1}(\tau) d \tau \\
& \frac{C_{2}(t)}{C_{2 p}(t)}=f-\frac{v_{1 e}}{C_{2 p}(t)}-f \frac{v_{1 p}}{v_{1 e}} \frac{\int_{0}^{t} C_{1 p}(\tau) d \tau}{C_{2 p}(t)}+v_{2 p}
\end{aligned}
$$




$$
\frac{C_{2}(t)}{C_{2 p}(t)}=f \frac{\int_{0}^{t} \alpha C_{1}(\tau) d \tau}{C_{2 p}(t)}-f \frac{v_{1 p}}{v_{1 e}} \frac{\int_{0}^{t} C_{1 p}(\tau) d \tau}{C_{2 p}(t)}+v_{2 p}
$$

At equilibrium in compartment $1, \mathrm{C}_{1 \mathrm{p}}(\mathrm{t})=\alpha \mathrm{C}_{1}(\mathrm{t})$ and $\mathrm{C}_{1 \mathrm{p}}(\mathrm{t})=\mathrm{C}_{2 \mathrm{p}}(\mathrm{t}), \boldsymbol{t}>\boldsymbol{t}_{\mathbf{1}}^{*}$

$$
\frac{C_{2}(t)}{C_{2 p}(t)}=f\left(1-\frac{v_{1 p}}{v_{1 e}}\right) \frac{\int_{0}^{t} C_{1 p}(\tau) d \tau}{C_{2 p}(t)}+v_{2 p} .
$$

Thus, the bias (overestimate) introduced into the estimate of flow from compartment 1 to compartment 2 is of the order of the ratio of the plasma to interstitial volume in compartment 1. In our example, this estimation error is $\sim 10 \%$. We note that the estimate produced by fitting the Patlak Plot of case 2 can be multiplied by $\frac{v_{1 e}-v_{1 p}}{v_{1 e}}$, thus producing an unbiased estimate of flow. However, this would add the errors in estimating $v_{1 p}$ and $v_{1 e}$ to the errors of estimating flow between the compartments. We have judged that a known bias of around $10 \%$ is acceptable for a stable estimate of intercompartmental flow. 
References

1. Hassid Y, Furman-Haran E, Margalit R, Eilam R, Degani H. Noninvasive magnetic resonance imaging of transport and interstitial fluid pressure in ectopic human lung tumors. Cancer Res 2006;66(8):4159-4166.

2. Hompland T, Ellingsen C, Rofstad EK. Preclinical evaluation of Gd-DTPA and gadomelitol as contrast agents in DCE-MRI of cervical carcinoma interstitial fluid pressure. BMC cancer 2012;12(1):544.

3. = Hompland T, Ellingsen C, Ovrebo KM, Rofstad EK. Interstitial fluid pressure and associated lymph node metastasis revealed in tumors by dynamic contrastenhanced MRI. Cancer Res 2012;72(19):4899-4908.

4. Liu LJ, Brown SL, Ewing JR, Schlesinger M. Phenomenological model of interstitial fluid pressure in a solid tumor. Physics Review E 2011;84(2):021919-021911 to 021919-021919.

5. Nield DA, Bejan A. Convection in Porous Media. 3rd edition. Springer Science, 2006. . New York, N.Y.: Springer Science+Business Media, Inc.; 2006.

6. Ewing JR, Bagher-Ebadian H. Model selection in measures of vascular parameters using dynamic contrast-enhanced MRI: experimental and clinical applications. NMR Biomed 2013;26(8):1028-1041.

7. Logan J. Graphical analysis of PET data applied to reversible and irreversible tracers. Nucl Med Biol 2000;27(7):661-670.

8. Logan J, Fowler JS, Volkow ND, Wolf AP, Dewey SL, Schlyer DJ, MacGregor RR, Hitzemann R, Bendriem B, Gatley SJ, et al. Graphical analysis of reversible radioligand binding from time-activity measurements applied to [N-11C-methyl]-()-cocaine PET studies in human subjects. J Cereb Blood Flow Metab 1990;10(5):740747.

9. Aryal MP, Nagaraja TN, Keenan KA, Bagher-Ebadian H, Panda S, Brown SL, Cabral G, Fenstermacher JD, Ewing JR. Dynamic contrast enhanced MRI parameters and tumor cellularity in a rat model of cerebral glioma at 7 T. Magn Reson Med 2014;71(6):2206-2214.

10. Tofts PS, Brix G, Buckley DL, Evelhoch JL, Henderson E, Knopp MV, Larsson HB, Lee TY, Mayr NA, Parker GJ, Port RE, Taylor J, Weisskoff RM. Estimating kinetic parameters from dynamic contrast-enhanced T(1)-weighted MRI of a diffusable tracêr: standardized quantities and symbols. J Magn Reson Imaging 1999;10(3):223232.

11. Aryal MP, Nagaraja TN, Brown SL, Lu M, Bagher-Ebadian H, Ding G, Panda S, Keenan K, Cabral G, Mikkelsen T, Ewing JR. Intratumor distribution and test-retest comparisons of physiological parameters quantified by dynamic contrast-enhanced MRI in rat U251 glioma. NMR Biomed 2014;27(10):1230-1238. 
12. Ewing JR, Brown SL, Lu M, Panda S, Ding G, Knight RA, Cao Y, Jiang Q, Nagaraja TN, Churchman JL, Fenstermacher JD. Model selection in magnetic resonance imaging measurements of vascular permeability: Gadomer in a 9L model of rat cerebral tumor. J Cereb Blood Flow Metab 2006;26(3):310-320.

13. Bagher-Ebadian H, Jain R, Nejad-Davarani SP, Mikkelsen T, Lu M, Jiang Q, Scarpace L, Arbab AS, Narang J, Soltanian-Zadeh H, Paudyal R, Ewing JR. Model selection for DCE-T1 studies in glioblastoma. Magn Reson Med 2012;68(1):241-251.

14. Gelman N, Ewing JR, Gorell JM, Spickler EM, Solomon EG. Interregional variation of longitudinal relaxation rates in human brain at $3.0 \mathrm{~T}$ : relation to estimated iron and = water contents. Magn Reson Med 2001;45(1):71-79.

15. Ewing JR, Nagaraja TN, Paudyal R, Bagher-Ebadian H, Knight RA, Ledbetter KA, Fenstermacher JD. An MRI Estimate of Vascular Permeability in 9L Cerebral Tumor Agrees with that of Quantitative Autoradiography. 2008; Toronto, Ca. Society of Magnetic Resonance in Medicine. p 1886.

16. Nagaraja TN, Aryal MP, Brown SL, Bagher-Ebadian H, Mikkelsen T, Yang JJ, Panda S, Keenan KA, Cabral G, Ewing JR. Cilengitide-induced temporal variations in transvascular transfer parameters of tumor vasculature in a rat glioma model: identifying potential MRI biomarkers of acute effects. PLoS One 2013;8(12):e84493.

17. Nagaraja TN, Karki K, Ewing JR, Divine GW, Fenstermacher JD, Patlak CS, Knight RA. The MRI-measured arterial input function resulting from a bolus injection of GdDTPA in a rat model of stroke slightly underestimates that of Gd-[(14)C]DTPA and marginally overestimates the blood-to-brain influx rate constant determined by Patlak plots. Magn Reson Med 2010;63(6):1502-1509.

18. Sourbron SP, Buckley DL. Classic models for dynamic contrast-enhanced MRI. NMR Biomed 2013;26(8):1004-1027.

19. Brown SL, Nagaraja TN, Aryal MP, Panda S, Cabral G, Keenan KA, Elmghirbi R, Mikkelsen T, Hearshen D, Knight RA, Wen N, Kim JH, Ewing JR. MRI-Tracked Tumor Vascular Changes in the Hours after Single-Fraction Irradiation. Epub before publication. Radiat Res 2015.

20. Scheffé H. The Analysis of Variance. Bradley RA, Hunter JS, Kendall DG, Watson GS, editors. New York, N.Y.: John Wiley \& Sons; 1959. 477 p.

21. Patlak CS, Blasberg RG, Fenstermacher JD. Graphical evaluation of blood-to-brain transfer constants from multiple-time uptake data. J Cereb Blood Flow Metab 1983;3:1-7.

22. Patlāk C, Blasberg R. Graphical Evaluation of blood to brain transfer constants from multiple time up take data. Generalizations. J Cereb Blood Flow Metab 1985;5:584590.

23. Pinheiro J, Bates DB, DebRoy SD, Sarkar D, Core Team R. nlme: Linear and Nonlinear Mixed Effects Models. R package 2013. 
24. Core Team R. A language and environment for statistical computing. Vienna, Austria: R Foundation For Statistical Computing; 2013.

25. Levin VA, Fenstermacher JD, Patlak CS. Sucrose and inulin space measurements of cerebral cortex in four mammalian species. Am J Physiol 1970;219(5):1528-1533.

26. Sykova E, Nicholson C. Diffusion in brain extracellular space. Physiological reviews 2008;88(4):1277-1340.

27. Munson JM, Shieh AC. Interstitial fluid flow in cancer: implications for disease progression and treatment. Cancer management and research 2014;6:317-328.

28. Pishko GL, Astary GW, Mareci TH, Sarntinoranont M. Sensitivity analysis of an = image-based solid tumor computational model with heterogeneous vasculature and porosity. Ann Biomed Eng 2011;39(9):2360-2373.

29. Boucher Y, Baxter LT, Jain RK. Interstitial pressure gradients in tissue-isolated and subcutaneous tumors: implications for therapy. Cancer Res 1990;50(15):44784484.

30. Pishko GL, Astary GW, Zhang J, Mareci TH, Sarntinoranont M. Role of convection and diffusion on DCE-MRI parameters in low leakiness KHT sarcomas. Microvasc Res 2012;84(3):306-313.

31. Sarntinoranont M, Rooney F, Ferrari M. Interstitial stress and fluid pressure within a growing tumor. Ann Biomed Eng 2003;31(3):327-335.

32. Netti PA, Berk DA, Swartz MA, Grodzinsky AJ, Jain RK. Role of extracellular matrix assembly in interstitial transport in solid tumors. Cancer Res 2000;60(9):24972503.

33. Pronin IN, McManus KA, Holodny AI, Peck KK, Kornienko VN. Quantification of dispersion of Gd-DTPA from the initial area of enhancement into the peritumoral zone of edema in brain tumors. J Neurooncol 2009;94(3):399-408.

34. Magdoom KN, Pishko GL, Rice L, Pampo C, Siemann DW, Sarntinoranont M. MRIbased computational model of heterogeneous tracer transport following local infusion into a mouse hind limb tumor. PLoS One 2014;9(3):e89594.

35. Sirianni RW, Zheng MQ, Saltzman WM, Huang Y, Carson RE. Direct, quantitative, and noninvasive imaging of the transport of active agents through intact brain with positron emission tomography. Molecular imaging and biology : MIB : the official publication of the Academy of Molecular Imaging 2013;15(5):596-605.

36. Pathak AP, Artemov D, Ward BD, Jackson DG, Neeman M, Bhujwalla ZM. Characterizing extravascular fluid transport of macromolecules in the tumor interstitium by magnetic resonance imaging. Cancer Res 2005;65(4):1425-1432.

37. Netti PA, Baxter LT, Boucher Y, Skalak R, Jain RK. Time-dependent behavior of interstitial fluid pressure in solid tumors: implications for drug delivery. Cancer Res 1995;55(22):5451-5458. 
38. Stylianopoulos T, Martin JD, Snuderl M, Mpekris F, Jain SR, Jain RK. Coevolution of solid stress and interstitial fluid pressure in tumors during progression: implications for vascular collapse. Cancer Res 2013;73(13):3833-3841.

39. Bruehlmeier M, Roelcke U, Blauenstein P, Missimer J, Schubiger PA, Locher JT, Pellikka R, Ametamey SM. Measurement of the extracellular space in brain tumors using 76Br-bromide and PET. J Nucl Med 2003;44(8):1210-1218.

40. Ludemann L, Grieger W, Wurm R, Budzisch M, Hamm B, Zimmer C. Comparison of dynamic contrast-enhanced MRI with WHO tumor grading for gliomas. Eur Radiol 2001;11(7):1231-1241.

41. = Yankeelov TE, Rooney WD, Li X, Springer CS. Variation of the Relaxographic "Shutter-Speed" for Transcytoliemmal Water Exchange Affects the CR BolusTracking Curve Shape. Magn Reson Med 2003;50(6):1151-1169.

42. Li X, Rooney WD, Varallyay CG, Gahramanov S, Muldoon LL, Goodman JA, Tagge IJ, Selzer AH, Pike MM, Neuwelt EA, Springer CS, Jr. Dynamic-contrast-enhanced-MRI with extravasating contrast reagent: rat cerebral glioma blood volume determination. J Magn Reson 2010;206(2):190-199.

43. Li X, Rooney WD, Springer CS, Jr. A unified magnetic resonance imaging pharmacokinetic theory: intravascular and extracellular contrast reagents. Magn Reson Med 2005;54(6):1351-1359.

44. Jain R, Ellika SK, Scarpace L, Schultz LR, Rock JP, Gutierrez J, Patel SC, Ewing J, Mikkelsen T. Quantitative estimation of permeability surface-area product in astroglial brain tumors using perfusion CT and correlation with histopathologic grade. AJNR Am J Neuroradiol 2008;29(4):694-700.

45. Blasberg RG, Fenstermacher JD, Patlak CS. Transport of alpha-aminoisobutyric acid across brain capillary and cellular membranes. J Cereb Blood Flow Metab 1983;3(1):8-32.

46. Bereczki D, Wei L, Otsuka T, Hans F-J, Acuff V, Patlak C, Fenstermacher J. Hypercapnia slightly raises blood volume and sizably elevates flow velocity in brain microvessels. American Journal of Physiology 1993;264:H1360-H1369.

47. Boucher Y, Salehi H, Witwer B, Harsh GRt, Jain RK. Interstitial fluid pressure in intracranial tumours in patients and in rodents. Br J Cancer 1997;75(6):829-836.

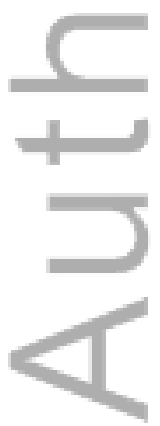

This article is protected by copyright. All rights reserved. 


\section{Peripheral Tissue Compression Predicts Exudate Flux in a Rat Model of Embedded Cerebral Tumor: an MRI Study}

James R. Ewing, Tavarekere N. Nagaraja, Madhava P. Aryal, Kelly A. Keenan, Rasha

Elmghirbi, Hassan Bagher-Ebadian, Swayamprava Panda, Mei Lu, Tom Mikkelsen,

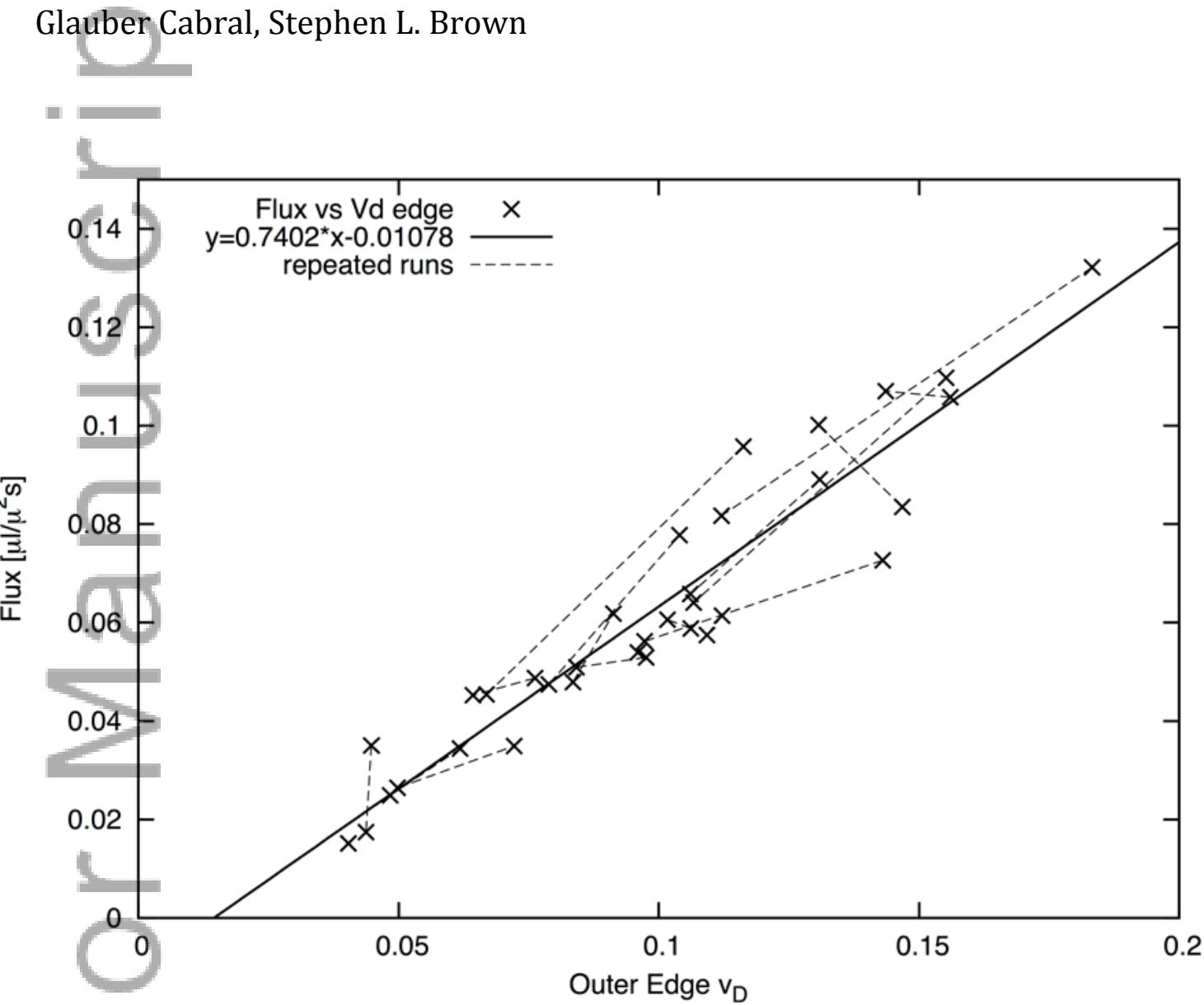

Exudate flux from an inner ring ROI (tumor) to an outer ring ROI (mostly normal tissue) versus the extracellular volume $\left(\mathrm{V}_{\mathrm{D}}\right)$ of the outer ring ROI in 18 athymic rats with an embedded U251 cerebral tumor. Sixteen of the studies were repeated in a 24 hour interval. The 16 repeated studies are connected with dashed lines. This very significant co-variance $\left(R^{2}=0.9\right)$ implies that a knowledge of $V_{D}$ in the rim of the will yield a remarkably precise prediction of exudate flow, regardless of other parameters such as TIFP and tumor porosity, which might otherwise be thought necessary for a prediction of the exudate flow rate. 